\title{
On the Impact of Long Wind-Waves on Near-Surface Turbulence and Momentum Fluxes
}

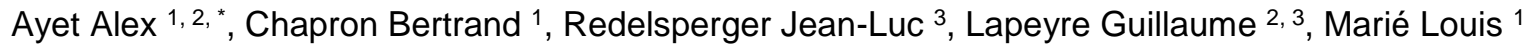

1 Univ. Brest, CNRS, IRD, Ifremer, Laboratoire d'Océanographie Physique et Spatiale (LOPS), IUEM, Brest, France

2 LMD/IPSL, CNRS, École Normale Supérieure, PSL Research University, Paris, France

3 Univ. Brest, CNRS, IRD, Ifremer, Laboratoire d'Océanographie Physique et Spatiale (LOPS), IUEM, Brest, France

* Corresponding author : Alex Ayet, email address : alex.ayet@normalesup.org

\begin{abstract}
:
We propose a new phenomenological model to represent the impact of wind-waves on the dissipation of turbulence kinetic energy near the sea surface. In this model, the momentum flux at a given height results from the averaged contribution of eddies attached to the sea surface whose sizes are related to the surface geometry. This yields a coupling between long wind-waves and turbulence at heights of about $10 \mathrm{~m}$. This new wind-and-waves coupling is thus not exclusively confined to the short wave range and heights below $5 \mathrm{~m}$, where most of the momentum transfer to the waves is known to occur. The proposed framework clarifies the impact of wind-waves on Monin-Obukhov similarity theory, and the role of long wind-waves on the observed wind-wave variability of momentum fluxes. This work reveals which state variables related to the wind-wave coupling require more accurate measurements to further improve windover-waves models and parametrizations.
\end{abstract}

Keywords : Air\{sea uxes, Wall-bounded turbulence, Wave boundary layer, Wind stress, Wind-waves 


\section{Introduction}

Observing a windy sea immediately reveals that wind and waves are strongly coupled. Yet, consistent physical

mechanisms explaining this two-way coupling are still elusive both to theory and observations (e.g. Soloviev and Kudryavtsev 2010; Hristov 2018; Villas Boas et al. 2019). Of particular interest is the link between nearsurface momentum fluxes and waves, due to its importance in atmospheric models, from the synoptic to the climate scale (e.g. Janssen and Viterbo 1996; Shimura et al. 2017; Pineau-Guillou et al. 2018; Villas Boas et al. 2019). For a given near-surface mean wind speed, a large source of variability in turbulent fluxes is atmospheric stability (e.g. Geernaert 1990; Fairall et al. 2003), consistently described by Monin-Obukhov Similarity Theory (MOST, see the review by Foken 2006). However, for neutral atmospheric conditions, open-ocean observations exhibit a variability around their mean value for a given mean wind speed, which has been attributed to waves (see e.g. Edson et al. 2013). The mean value results from a local equilibrium between short wind-waves and atmospheric turbulence. For low wind speeds, swell and non-stationary wind conditions have been suggested as possible reasons of its variability (Drennan et al. 1999), whereas at moderate to high wind speeds, the physical processes are still not clearly determined.

Close to the surface, wave impact on atmospheric turbulence has been accounted for through the so-called wave-induced stress. Assuming that wind fluctuations can be described as a linear superposition of a turbulent and an ocean-wave induced component, waveinduced stress results, for growing seas, from the transfer of mean flow energy to the wave-induced component (Janssen 1989). This energy is then transfered to turbulent motions, which support the growth of windwaves (Plant 1982). In an equilibrium wind-over-waves situation, i.e. for waves that equilibrated with a local stationary airflow, wave-induced stress induces an en- 
hancement of turbulent motions compared to flow over a smooth surface (Makin and Mastenbroek 1996). This net enhancement occurs up to a height that defines the wave boundary layer (WBL), above which waveinduced stress vanishes. Wave-induced stress is mostly correlated to the presence of short wind-waves, which are thus strongly coupled to the low-level wind field and receive most of the wind energy input. Conceptual models including this physical process were able to successfully predict measured open-ocean fluxes (Makin and Kudryavtsev 1999; Hara and Belcher 2002; Kudryavtsev et al. 2014). These single-column models (called wind-over-waves models in the following) couple a turbulence kinetic energy (TKE) equation to a spectral wave model through wave-induced stress, and predict the equilibrated turbulent momentum flux and wind-wave spectrum given a reference-height mean wind speed. Following experimental and numerical studies, Kudryavtsev et al. (2014) included wave-breaking effects (i.e. discontinuities in the surface slope) as an additional source of wave-induced stress, and showed that this effect could be significant in explaining the observed momentum fluxes. Being mostly supported by short waves (with wavelength of the order of 0.01 to 1 metres), both processes act on a shallow atmospheric layer of height one order of magnitude smaller than their wavelength. This results in a height of the WBL of the order of $5 \mathrm{~m}$ in the absence of swell.

While wave-induced contributions to atmospheric variables are often reported as being particularly difficult to detect at higher altitudes from single-point measurements (Soloviev and Kudryavtsev 2010), Edson et al. (2004) mention that "field campaigns have shown that some turbulent statistics, e.g., the pressure transport term in the kinetic energy budget equation, are influenced by waves up to heights where $k_{p} z \sim 2$, where $k_{p}$ is the peak wavenumber of the dominant waves. The latter findings suggest a thicker WBL for some characteristics of the flow". Hence the coupling between atmospheric turbulence and wind-waves could possibly extend on vertical scales much above $5 \mathrm{~m}$, suggesting the existence of other processes beyond wave-induced stress. Those processes, as wave-induced stress, result from spatial correlations between atmospheric quantities on the scale of wind-waves, and should thus be more easily observed if spatial statistics of the atmospheric field (e.g., multiple-point measurements) are available. In the absence of such measurements, a method extracting those spatial correlations from single-point measurements is necessary (as developed for wave-induced stress in Hristov et al. 1998, 2003). The present study is a first step towards such a method by revealing state variables in which those processes might be buried.
More generally, the understanding of the local windand-waves equilibrium is related to the longstanding question of the influence of a structured boundary (both in terms of geometry and of velocity) on the properties of turbulence at a certain distance from the boundary (see the review by Belcher and Hunt 1998). Waveinduced stress only accounts for the interaction of the turbulent field with additional, wave-induced, fluctuations. It does not represent the possible reorganization of the turbulent fluctuations due to the presence of a structured boundary (e.g. the formation of rolls presented by Phillips et al. 1996). This reorganization has been shown to occur due to stratification effects for a flat and non-moving boundary (in experiments, theory, and numerical simulations respectively: Kaimal et al. 1972; Elperin et al. 2002; Li et al. 2018).

A phenomenological model enabling the inclusion of organized turbulent structures near a wall has been recently described in Gioia et al. (2010). The model assumes that the turbulent fluxes at a given height are driven by surface-attached eddies representing crosswind atmospheric turbulent structures in a convected frame of reference (invariant in the spanwise direction), and whose horizontal and vertical length scales are related to the height at which the flux is computed (dashed line in Fig. 1). The reorganization of these attached eddies due to stratification was then included in this model by Katul et al. (2011) and Li et al. (2012). These authors introduced an "eddy anisotropy" coefficient $f_{a}$ (related to the eddies horizontal to vertical aspect ratio), accounting for the deformation of attached eddies due to buoyancy forces (Fig. 1a). As this deformation can be linked to properties of the turbulence spectra (as explained in Katul and Manes 2014), the authors calibrated eddy anisotropy based on measurements from Kaimal et al. (1972). One of the main outcomes of Katul et al. (2011) is to recover MOST universal functions, which were obtained through measurements, using a theoretical model based on a TKE balance. In this balance, the deformation of attached eddies translated into a change in TKE dissipation.

In the presence of surface waves, the link between the shape and spatial organization of the turbulent structures and the geometry of the surface is still an open question. Hence, we (i) propose that wind-waves deform attached eddies, inducing a change in TKE dissipation; (ii) model this effect within the Katul et al. (2011) framework; and (iii) evaluate the impact of this deformation on turbulent momentum fluxes, and its ability to explain the observed variability at moderate wind speeds. The model assumes that the deformed surface allows attached eddies of different sizes to contribute to the momentum flux at a given height (Fig. 1b). The pro- 

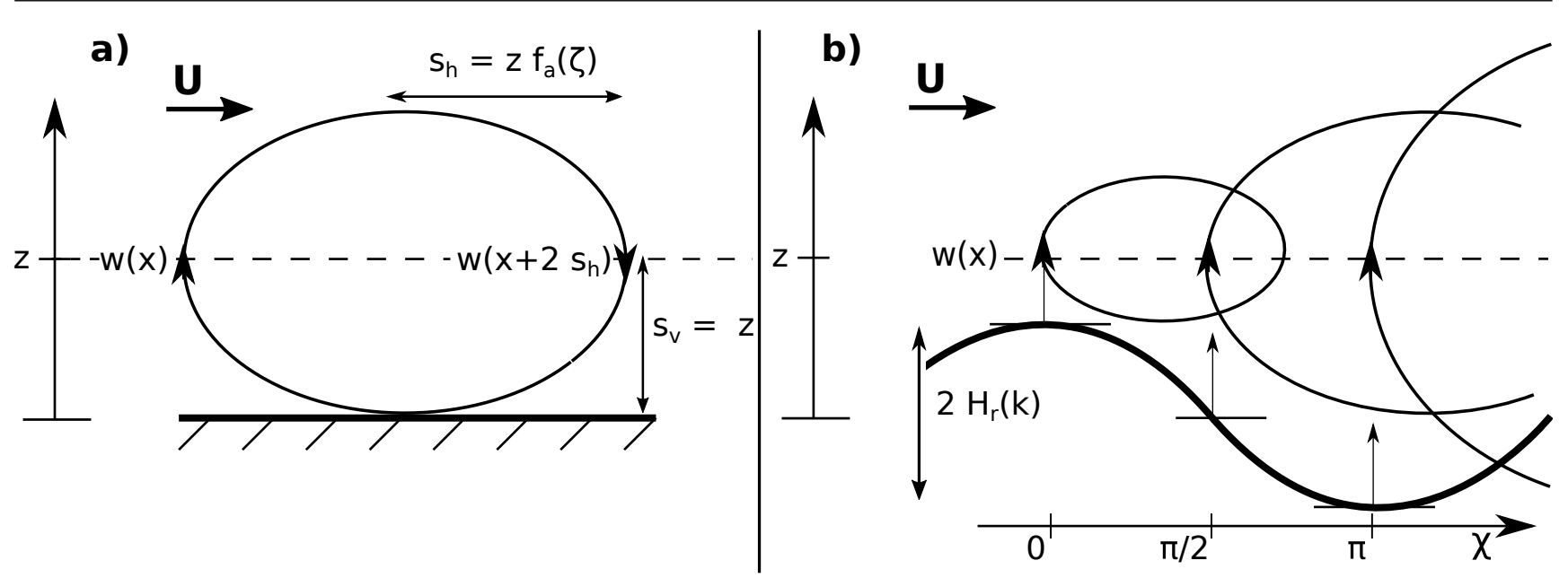

Fig. 1 (a) State-of-the-art and (b) proposed attached-eddy model describing vertical turbulent fluxes through a surface at a height $z$ (dashed lines). (a) For wall-bounded turbulence (Gioia et al. 2010), the most energetic structure (i.e. inducing most of the fluxes) has a vertical length scale $2 s_{v}$ equal to twice the considered height $z$. Its horizontal length scale $2 s_{h}$ varies with stratification (Katul et al. 2011). The difference between the upward and downward vertical velocity of the structure $w$ yields the mean vertical turbulent motion. The structure can be notionally represented by an attached eddy (thin solid line). (b) In the presence of a surface wave (thick solid line), depending on the relative phase between the eddy and the wave (denoted $\chi$ ), the height of the surface and thus the size of the attached eddy varies. We propose that multiple attached eddies contribute to the flux (only three examples are drawn)

posed physical mechanism is mainly supported by long wind-waves (wavelengths of the order of $10 \mathrm{~m}$ ), and results in a modification of TKE dissipation at heights above $5 \mathrm{~m}$. This mechanism introduces a dependency of the wind-and-waves local equilibrium to the local spectral characteristics of long wind-waves and to the intensity of the modulation mechanism. This variability is then used to explain open-ocean measurements using the wind-over-waves model of Kudryavtsev et al. (2014) to obtain the mean observed wind-and-waves equilibrium.

The paper is organized as follows: the wall-bounded model and the new physical mechanism are presented in Sects. 2 (for a single wind wave) and 3 (for a realistic sea surface). The wind-over-waves model is briefly summarized in Sect. 4. Section 5 focuses on the resulting impact of the coupling mechanism on near-surface momentum fluxes for neutral conditions, allowing explanation of their variability for a given wind in open ocean measurements. Section 6 then studies the effect of stability on near-surface turbulence, by linking the model with MOST and comparing it to measurements. Conclusions are presented in Sect. 7.

\section{A Wall-Bounded Turbulence Model over a Monochromatic Wave}

In this section, we propose a new mechanism to model the impact of a wave with a specific wavenumber on TKE dissipation. To this end, starting from a model de- veloped to describe wall-bounded stratified turbulence (recalled in Sect. 2.1), an extension is proposed in order to account for a periodic and undulating surface (Sect. 2.2).

\subsection{A Model for Wall-Bounded Stratified Turbulence}

We first recall the framework presented in Gioia et al. (2010), Katul et al. (2011) and Li et al. (2012) to describe a stratified surface boundary layer (SBL). The framework models the SBL by means of a TKE balance equation. The mean wind shear and stratification are specified, and the model predicts turbulent fluxes. The key result of the framework is to derive a closure for TKE dissipation by considering the shape of eddies attached to the surface.

The SBL is defined as the lowest part of the surface atmospheric boundary layer (adjacent to the surface) where the flow is horizontally homogeneous and stationary, and with no subsidence. In what follows, the dominant wind-waves are assumed to be aligned with the mean wind direction (and the horizontal coordinate $x$ ), so that we only consider perturbations in the $(x, z)$ directions (where $z$ is the vertical coordinate). The turbulent momentum flux normalized by air density $\left(u_{*}^{l}\right)^{2}=-\overline{u^{\prime} w^{\prime}}$ is constant within the layer due to horizontal homogeneity. Anticipating Sect. $4, u_{*}^{l}$ is called the local friction velocity.

The TKE balance equation within this layer is assumed to be a balance between mechanical (or shear) 
production, buoyancy production/destruction, and TKE dissipation $\epsilon$

$-\overline{u^{\prime} w^{\prime}} \frac{\partial U}{\partial z}+\frac{g}{\theta_{h}} \overline{w^{\prime} \theta^{\prime}}=\epsilon$,

where $U$ is the mean wind speed and $(\cdot)^{\prime}$ denotes turbulent fluctuations. In the following, water vapour effects are omitted for the sake of simplicity. Defining $H$, the sensible heat flux within the SBL, as

$H=-\rho C_{p} \overline{w^{\prime} \theta^{\prime}}$,

and using the definition of $u_{*}^{l}$, the TKE balance can been written as

$\left(u_{*}^{l}\right)^{2} \frac{\partial U}{\partial z}-\frac{g H}{\rho C_{p} \theta_{h}}=\epsilon$,

where $g$ is the acceleration due to gravity, $\rho$ isair density, and $C_{p}$ is the heat capacity of dry air.

We also introduce the Obukhov length,

$L=\frac{\rho C_{p} \theta_{h}\left(u_{*}^{l}\right)^{3}}{\kappa g H}$

where $\kappa=0.4$ is the von Kármán constant and $\theta_{h}$ is a reference potential temperature. With this definition, the stability parameter $\zeta$ is defined as $\zeta=z / L$, being negative for an unstable boundary layer and positive in the stable case.

The TKE balance equation can then be rewritten in dimensionless form from Eqs. 2 and 3, and the definition of $\zeta$ (see e.g. Hogstrom 1996)

$-\frac{\kappa z}{u_{*}^{l}} \frac{\partial U}{\partial z}+\zeta+\frac{\kappa z}{\left(u_{*}^{l}\right)^{3}} \epsilon=0$.

Following Katul et al. (2011), we further include the first-order effect of the turbulent flux-transport and pressure redistribution terms (which are neglected in Eq. 1, and were shown to be significant for non-neutral conditions) as a constant correction $\beta_{2}$ to the buoyancy term, yielding

$-\frac{\kappa z}{u_{*}^{l}} \frac{\partial U}{\partial z}+\left(1+\beta_{2}\right) \zeta+\frac{\kappa z}{\left(u_{*}^{l}\right)^{3}} \epsilon=0$,

where $\beta_{2}=1$ (Katul et al. 2011).

To solve this equation (i.e. to obtain $u_{*}^{l}$ from given values of $\partial U / \partial z, z$, and $\zeta$ ), a closure for TKE dissipation $\epsilon$ is necessary. To this end, Gioia et al. (2010) and Katul et al. (2011) proposed that turbulent fluxes at a height $z$ are determined by the mean difference between vertical velocities $w$ at $x$ and $x+2 s_{h}$ (at the same height $z$ ) corresponding to the edges of a turbulent structure with given horizontal $\left(2 s_{h}\right)$ and vertical $\left(2 s_{v}\right)$ length scales. In such a situation, the momentum flux was estimated as

$$
\begin{aligned}
\left(u_{*}^{l}\right)^{2}(z) & =\kappa_{T}\left|w\left(x+2 s_{h}\right)-w(x)\right|\left[U\left(z+s_{v}\right)-U\left(z-s_{v}\right)\right] \\
& \sim \kappa_{T}\left|w\left(x+2 s_{h}\right)-w(x)\right| \frac{\partial U}{\partial z} 2 s_{v},
\end{aligned}
$$

i.e., as the product between the turbulent structure mean vertical velocity and the horizontal momentum perturbation, assuming that momentum is transported across the entire vertical extension of the structure (Gioia et al. 2010). In the above expression, $\kappa_{T}$ is a dimensionless proportionality coefficient.

The vertical velocity differences were then estimated using the Kolmogorov 4/5 law for the third-order velocity structure function (e.g. Monin and Yaglom 1975),

$\left|w\left(x+2 s_{h}\right)-w(x)\right|=\left(\kappa_{\epsilon} \epsilon s_{h}\right)^{1 / 3}$,

where $\kappa_{\epsilon}$ is a dimensionless proportionality coefficient. Inserting (7) into (6) and after some algebra, the following expression results for dissipation,

$\epsilon=\kappa^{-4}\left(u_{*}^{l}\right)^{6}\left(\frac{\partial U}{\partial z}\right)^{-3} s_{v}^{-3} s_{h}^{-1}$,

where $\kappa=2^{3 / 4} \kappa_{T}^{3 / 4} \kappa_{\epsilon}^{1 / 4}$ is the von Kármán constant (this matching is required to recover the law-of-the-wall under neutral conditions).

In Eqs. 6-8 only the most energetic structure at a height $z$ is considered, i.e. corresponding to the leading order contribution to the third-order structure function and hence to the vertical momentum flux. It is the structure whose half vertical length scales as the height at which the turbulent flux is computed, i.e. $s_{v}=z$. In the absence of stratification, the horizontal length scale is further assumed to be equal to the vertical length scale (Gioia et al. 2010). Katul et al. (2011) showed that stratification introduces an eddy anisotropy factor $f_{a}$ in the horizontal length scale, such that

$s_{h}=f_{a}(\zeta) s_{v}$,

where $f_{a}=1$ for neutral conditions (i.e. $\zeta=0$ ).

Figure 1a shows a conceptual representation of the most energetic turbulent structure for a height $z$ as an ensemble-mean eddy in a convected frame of reference (following Gioia et al. 2010; Katul et al. 2011). The shape of the eddy depends on the horizontal and vertical length scales of the turbulent structure. This conceptual representation does not entail any velocity field associated with the eddy, apart from the vertical velocities at its upward and downward branches (black arrows), corresponding to $w(x)$ and $w\left(x+2 s_{h}\right)$ respectively.

The condition $s_{v}=z$ then yields that the eddies associated with the most energetic turbulent structures 
(termed energy-containing eddies) are eddies attached to the surface, reminiscent of the attached-eddy model of turbulence introduced by Townsend (1980) (see also the review by Marusic and Monty 2019). The spatial aspect ratio of the energy-containing eddies, fixed by relation (9), reflects their reorganization due to buoyancy forces (through the stability parameter $\zeta$ ).

\subsection{Inclusion of a Single Wave within the Wall-Bounded Model}

We now propose an extension of the wall-bounded model to include the reorganization of energy-containing eddies due to a boundary with a spatial structure. Let us first consider the impact of a wave of wavenumber $k$ and of height $H_{r}(k)$ on an attached eddy driving the momentum flux at a height $z$. In the following, we derive the horizontal extent of the eddy $2 \tilde{s}_{h}$ in the presence of the monochromatic wave. More generally, we use the notation $\widetilde{(\cdot)}$ throughout to denote the contribution a single wave of wavelength $k$ to turbulent quantities.

The sea-surface height $h$ varies around its reference value depending on the position along the wave, measured by the relative phase (denoted $\chi$ ) between the wave and the eddy. The height variations follow $h(k, \chi)=H_{r}(k) \cos \chi$. Within the wall-bounded model presented in Sect. 2.1, the vertical extent of the most efficient eddy is twice the distance between the surface and the height $z$ (Fig. 1a). In the presence of a surface wave, the now phase-dependent vertical extent of the attached eddy driving the momentum flux $\tilde{s}_{v}$ varies around its reference value $z$. Figure $1 \mathrm{~b}$ shows the configurations corresponding to $\chi=0, \pi / 2$, and $\pi$.

Using (9), the horizontal length scale of the eddy varies as a function of phase $\chi$ as

$$
\begin{aligned}
\tilde{s}_{h}(z, \zeta, k, \chi) & \left.=f_{a}(\zeta)(z-h(k, \chi))\right) \\
& =z f_{a}(\zeta)\left[1-\left(H_{r}(k) / z\right) \cos \chi\right] .
\end{aligned}
$$

For a given wave, we further consider only the "outer region" of the SBL (as defined in Belcher and Hunt 1993, 1998). Within this region, (i) the mean flow speed is larger than the phase speed of the wave, and (ii) eddies have a turnover time longer than the advection time above the considered wave. Thus, during the eddy lifetime and its advection above a wave, different configurations (depending on $\chi$, and shown in Fig. 1b) can occur, which can all potentially contribute to the resulting upward transport of momentum (Eq. 6) and to TKE dissipation (Eq. 8).

We consider the most general form accounting for the contribution of all possible configurations to the eddy horizontal length scale, a weighted average over all configurations

$\left\langle\tilde{s}_{h}\right\rangle(z, \zeta, k)=\int_{0}^{\pi} p(\chi) \tilde{s}_{h}(z, \zeta, k, \chi) d \chi$,

where $p(\chi)$ is a weight, or the (normalized) probability density function (p.d.f.) of the configurations, and $\langle\cdot\rangle$ denotes the average over all configurations labeled by $\chi$. The average vertical extent of the attached eddy is assumed to be unchanged by the presence of waves (i.e. $\left\langle\tilde{s}_{v}\right\rangle=s_{v}=z$ ). From Eq. 6 (see also Gioia et al. 2010), the vertical extent results from the Taylor expansion of vertical wind variations (i.e. $U\left(z+s_{v}\right)-U(z-$ $\left.\left.s_{v}\right) \sim(\partial U / \partial z) 2 s_{v}\right)$ and denotes the height over which the eddy mixes momentum. The horizontal extent denotes the size and energy of the structure (through Kolmogorov's law, Eq. 7). We thus consider that, on average, the presence of waves only affects the energy of the horizontal structure.

If the p.d.f. $p(\chi)$ is not symmetric around $\chi=\pi / 2$, then the wave-induced sea-surface height variation leads on average to a variation of the eddy aspect ratio, affecting TKE dissipation (Eq. 8). In particular, a compression (respectively a stretching) occurs for a distribution where configurations around $\chi=0$ (resp. $\chi=\pi$ ) are dominant.

The different configurations in $\chi$ can also be interpreted as representing TKE bursts. From Kolmogorov's law (Eq. 7), a change in $s_{h}$ is related to a change in the vertical velocity difference of the turbulent structure. Increase or decrease in $s_{h}$ due to a change in the phase $\chi$ can thus be interpreted as a increase or decrease in the vertical velocity at the edges of the turbulent structure, and those variations associated with bursts. The average horizontal length scale computed in Eq. 11 can thus be interpreted as accounting for the contribution of bursts to TKE dissipation in the SBL due to the presence of waves. Bursts were suggested as possibly supporting a large fraction of open-ocean surface momentum fluxes (Dorman and Mollo-Christensen 1973). Laboratory measurements revealed that such events could have an asymmetric p.d.f. (see Fig. 26 of Kawamura and Toba 1988).

\section{The Impact of Wind-Waves on Near-Surface Turbulent Structures}

Section 2 presented a wall-bounded turbulence model where the effects of a single wave were included in TKE dissipation through the stretching or the compression of an attached eddy, i.e. the change in its horizontal to vertical aspect ratio. We now generalize the model to the case of a wave field (Sect. 3.1), while Sect. 3.2 
then discusses the physical quantities modulating the proposed mechanism.

3.1 Generalization of the Eddy-Stretching Process to a Wind-Wave Sea

So far we discussed how a monochromatic wave could impact a given turbulent structure. In the case of a wave field composed of the sum of monochromatic waves of different wavelengths and directions the question is to determine which waves can potentially stretch or compress an attached eddy contributing to the momentum flux at a height $z$ (in the sense of changing its aspect ratio). In the following, we make the assumption that the deformation occurs mainly when wave and attached eddy sizes are close, i.e. for a wave of wavelength $k$ scaling as the inverse of the height $1 / z$, with $z$ roughly the horizontal extent of the eddy (defined in Eq. 10).

The assumption can be made more precise by considering the physical mechanisms likely to cause eddy deformation. The geometry of short wind-waves is modulated by the supporting longer waves. Hence, the resulting surface roughness (due to wave-induced stress from short wind-waves) varies horizontally following the longer waves, on lengths of half the modulating wave horizontal length scale (e.g. Kudryavtsev and Chapron 2016). This modulation has been shown to significantly affect the near-surface atmospheric flow (Gent and Taylor 1976; Kudryavtsev and Chapron 2016) and could also impact attached eddies by inducing a roughness variation on a scale resonant with that of the eddy. The average deformation of an attached eddy by a wave, described in Sect. 2.2, is thus assumed to be due to these modulated shorter waves. Within this picture, modulating waves whose half horizontal length scale is shorter than the horizontal eddy size are not capable of interacting with both the upward and the downward branch of the attached eddy. Moreover, among these longer modulating wind-waves, we only consider the one experiencing the longest interaction time with the eddy advected above, i.e. the shortest (fastest) wind wave. It is thus assumed that eddy stretching or compression occurs for a resonant wave whose half-horizontal extent $\left(\pi / k_{r}\right)$ is equal to the horizontal length scale of the wall-bounded attached eddy prior to deformation $\left(2 z f_{a}\right)$

$k_{r}(z)=\frac{\pi}{2 z f_{a}(\zeta)}$.

The horizontal extent of an energy-containing eddy at a height $z$ over a wave field, $2\left\langle s_{h}\right\rangle$, is then expressed from the individual contribution of monochromatic waves as

$\left\langle s_{h}\right\rangle(z, \zeta)=\left\langle\tilde{s}_{h}\right\rangle\left[z, \zeta, k_{r}(z)\right]$.
Using Eqs. 10 and Eq. 11, it further reads

$\left\langle s_{h}\right\rangle(z, \zeta)=z f_{a}(\zeta) g_{e}(z, \zeta)$,

where we defined the eddy-stretching factor $g_{e}$ as

$g_{e}(z, \zeta)=\int_{0}^{\pi}\left\{1-\frac{H_{r}}{z} \cos \chi\right\} p(\chi) d \chi$

where $H_{r}$ is the height of the resonant wave.

For a sea surface described by a wave spectrum $S(k)$ (as a function of the isotropic wavenumber $k$ ), $H_{r}$ can be computed from contributions of a narrow wave-packet around $k_{r}$, of width $\Delta k$ as

$H_{r}^{2}\left(k_{r}\right)=\int_{k_{r}-\Delta k / 2}^{k_{r}+\Delta k / 2} S(p) d p \approx S\left(k_{r}\right) \Delta k$.

Note that for the physical picture of Fig. 1 to hold, the resonant wave height must be lower than the height $z$ at which fluxes are computed, restricting the physical process to cases where $H_{r}\left(k_{r}\right)<z$.

The width of the wavepacket $\Delta k$ is related to the accuracy of the resonance condition (Eq. 12), since it quantifies to what extent waves that are not exactly of wavelength $k_{r}$ contribute to eddy stretching. It is thus related to the magnitude of the physical mechanism causing eddy stretching, i.e. the modulation of short wind-waves by long wind-waves, which is highly variable (e.g. due to slicks, sea surface temperature, and jointly varying surface currents and stability conditions, see Vandemark et al. 1997; Grodsky et al. 2012; Kudryavtsev et al. 2012). The wave-packet width is thus considered as a model parameter, called $\Delta_{k}$. The resulting form for the wave-packet width, including the physical condition mentioned above is

$\Delta k=\left\{\begin{array}{ll}\Delta_{k} & \text { for } H_{r}\left(k_{r}\right)<z \\ 0 & \text { for } H_{r}\left(k_{r}\right) \geq z\end{array}\right.$.

For $H_{r}\left(k_{r}\right) \geq z$, or for no waves (i.e. $S(k)=0$ ), Eq. 14 is reduced to the expression proposed by Katul et al. (2011) (Eq. 9).

Eddy stretching accounts for the change in the shape of an energy-containing eddy by interaction with a surface wave of a size resonant with the size of the eddy. Using Eq. 14 in Eq. 8, TKE dissipation including eddy stretching reads

$\frac{\kappa z}{\left(u_{*}^{l}\right)^{3}} \epsilon=\frac{\left(u_{*}^{l}\right)^{3}}{(\kappa z)^{3}}\left(\frac{\partial U}{\partial z}\right)^{-3} f_{a}(\zeta)^{-1} g_{e}(z, \zeta)^{-1}$.

Due to the resonance condition between the wave and the eddy (Eq. 12), the change in TKE dissipation due to eddy stretching only occurs at heights $h_{e}=\pi /\left[2 f_{a}(\zeta) k\right] \sim$ $1 / k$, matching the heights suggested in Edson et al. (2004) and also discussed in the introduction. 
For $g_{e}$ and $f_{a}$ equal to one (i.e. neutral conditions and a flat boundary), we recover the expression of TKE dissipation obtained for homogeneous and isotropic turbulence (and used in the wind-over-waves model of Kudryav sev et al. 2014, see Sect. 4).

\subsection{Sources of Variability of Eddy Stretching}

Eddy stretching is a new coupling mechanism between the wave and the wind fields, whose magnitude can vary for a given mean wind speed. In order to understand the sources of this variability, the expression of eddy stretching presented in Eq. 15 is rewritten in wavenumber space, i.e. by defining $\tilde{g}_{e}$ such that

$g_{e}(z, \zeta)=\tilde{g}_{e}\left(k_{r}, \zeta\right)$.

By further using the resonance condition (Eq. 12) and the expression of the height of the resonant wave (Eqs. 16 and 15) yields

$\tilde{g}_{e}\left(k_{r}, \zeta\right)=1-2 \Delta k^{1 / 2} \frac{f_{a}(\zeta)}{\pi}\left[k_{r}^{2} S\left(k_{r}\right)\right]^{1 / 2} \int_{0}^{\pi} p(\chi) \cos \chi d \chi$.

In addition to stability, $\tilde{g}_{e}$ depends on the wavenumber of the resonant wave $\left(k_{r}\right)$ through the spectrum of the wave slopes $\left(k_{r}^{2} S\left(k_{r}\right)\right)$. Typical wind-wave-slope spectra exhibit a peak depending on the degree of seastate development (e.g. spatial fetch, as modelled in Donelan et al. 1985; Elfouhaily et al. 1997), and almost vanish for waves below $1 \mathrm{~m}$ (corresponding to $k_{r} \sim 10$ $\mathrm{m}^{-1}$ ). For waves larger than the spectral peak (e.g. 60 $\mathrm{m}$ for a fetch of $100 \mathrm{~km}$ in the Donelan et al. 1985, model), $k_{r}^{2} S\left(k_{r}\right)$ quickly vanishes. Eddy stretching thus reflects the impact of intermediate to long wind-waves (of the order of tenths of metres) on atmospheric turbulence, through the increase of the air-sea interface area (related to the sea surface mean slope). This is consistent with remote sensing measurements indicating a sensitivity of air-sea fluxes to the air-sea interface area (similar to radar backscatter, e.g. Kitaigorodskii 1973; Brown 1979; Vandemark et al. 1997). The range of the spectrum contributing the most to sea-surface slope (long wind-waves) can be highly variable for a given 10$\mathrm{m}$ mean wind speed, sensitive to fetch, rising/decaying winds, surface currents, modulating longer swells, thus introducing variability into the wind-and-waves equilibrium (e.g. see Zhang et al. 2009, where surface currents caused long wind-waves to deviate from the mean wind direction, impacting surface stress).

Eddy stretching also depends on the probability distribution of the different events $p(\chi)$ and the bandwidth coefficient $\Delta_{k}$. Variations of $p(\chi)$ can induce an eddy stretching smaller or greater than one. As shown in Fig. 1, for probability distributions where predominant configurations are for phases smaller than $\pi / 2$, the horvizontal extent of the eddy is reduced with respect to the wall bounded case, and hence eddy stretching is smaller than one (see Eq. 14). Conversely, when predominant configurations are for phases greater than $\pi / 2$, eddy stretching is larger than one. Both quantities $p(\chi)$ and $\Delta_{k}$ are related to the physical process inducing eddy stretching (the modulation of short wind-waves stress by long wind-waves) whose magnitude can vary for a given 10-m mean wind (Gent and Taylor 1976; Dulov et al. 2013; Kudryavtsev and Chapron 2016).

For a given 10-m mean wind speed, eddy stretching can thus vary due to (i) variations of the waveslope spectrum, and (ii) variation of the magnitude of the physical process causing eddy stretching. However, as described in Sect. 4, in order to obtain a realistic wind-wave spectrum and the associated windover-waves equilibrium (i.e. matching observations), we use the wind-over-waves model of Kudryavtsev et al. (2014). Within this particular model, the only parameter controlling the long wind-wave spectrum is spatial fetch, following the parametrization of Donelan et al. (1985). To simply account for deviations from this parametrization, as well as for the sources of variability mentioned above, Eq. 20 is rewritten as

$\tilde{g}_{e}\left(k_{r}, \zeta\right)= \begin{cases}1-\gamma \frac{f_{a}(\zeta)}{\pi}\left[k_{r}^{2} S\left(k_{r}\right)\right]^{1 / 2} & \text { for } H_{r}\left(k_{r}\right)<z \\ 1 & \text { for } H_{r}\left(k_{r}\right) \geq z\end{cases}$

where

$\gamma=2 \Delta_{k}^{1 / 2} \int_{0}^{\pi} p(\chi) \cos \chi d \chi$

The new parameter $\gamma$ contains all the dependencies to $p(\chi)$ and $\Delta_{k}$. Implicitly, it also contains variations in the wind-wave slope spectrum not described by the wind-over-waves model (e.g. non-stationary winds, surface currents, etc.).

Variations of spatial fetch in the Donelan et al. (1985) parametrization only change the spectrum of wind-waves greater than about $60 \mathrm{~m}$ (by causing a shift of the peak of the wind-wave slope spectra towards larger waves). From Eq. 21, this induces a change in eddy stretching at heights $h_{e}$ (proportional to the wave size) too large to impact the surface momentum flux $u_{*}$ (not shown). On the other hand, variations of $\gamma$ in Eq. 21 induce a global change of eddy stretching magnitude. This includes a change for eddy stretching magnitude corresponding to $10-\mathrm{m}$ waves, describing a change in the waves energy 
not described by the Donelan et al. (1985) parametrization. This corresponds to changes in eddy stretching at heights around $10 \mathrm{~m}$ having a significant impact on momentum fluxes as will be shown numerically in Sect. 5 .

The condition $H_{r}\left(k_{r}\right)<z$ in Eq. 21 sets upper and lower bounds on $\tilde{g}_{e}$ : since $H_{r}\left(k_{r}\right)$ is related to $k_{r}^{2} S\left(k_{r}\right)$, this condition sets an upper bound on $k_{r}^{2} S\left(k_{r}\right)$, implying that $\tilde{g}_{e}$ cannot be too large or too small relative to one for $\gamma<0$ or $\gamma \geq 0$ respectively. In practice, evaluating this condition would require evaluating $H_{r}\left(k_{r}\right)$ through Eq. 16, and hence choosing a value for $\Delta_{k}$. This is not compatible with the choice, made in this work, to use only one free parameter in the description of eddy stretching (the parameter $\gamma$ ). Hence, in the following, the condition $H_{r}\left(k_{r}\right)<z$ is replaced by setting bounds on $g_{e}$, i.e. by the condition that $\tilde{g}_{e}<10^{2}$ or $\tilde{g}_{e}>10^{-2}$ for $\gamma<0$ or $\gamma \geq 0$, respectively. This choice of bounds covers two orders of magnitude of $\tilde{g}_{e}$ around one. It also ensures that $\tilde{g}_{e}$ is positive, and hence that the TKE dissipation is positive.

\section{Wind-over-Waves Model}

In the previous sections, we proposed a mechanism accounting for the impact of long wind-waves on TKE dissipation within an SBL model where a wave spectrum was prescribed. We now briefly describe the wind-overwaves model introduced in Kudryavtsev et al. (2014), predicting the generation of wind-waves by turbulent motions within a wind-and-waves equilibrium. This windover-waves model is then used in the following sections to explore the sensitivity of the wind-and-waves equilibrium to the proposed mechanism.

The wind-over-waves model couples an atmospheric TKE equation with an equation describing a wind--wave field. Low-level turbulent motions lose energy to short wind-waves, which in turn generate atmospheric fluctuations enhancing TKE by extracting energy from the mean flow. Wave-wave non-linear interactions then result in an equilibrium wind-and-waves state, where TKE is enhanced with respect to flow over a smooth surface, reproducing the mean observed momentum flux in open-ocean measurements for a given mean wind under neutral conditions. At the core of this coupling is thus the transfer of energy between atmospheric turbulent motions and atmospheric wave-induced motions, the latter being coupled to the wind-wave field (e.g. Makin and Kudryavtsev 1999; Hara and Belcher 2002; Kudryavtsev et al. 2014). The atmospheric flow is thus decomposed into a mean component, a turbulent component and a wave-induced component which decays with height.
The first implication of this triple decomposition is that, as opposed to a standard SBL, the turbulent momentum flux $-\overline{u^{\prime} w^{\prime}}=\left(u_{*}^{l}\right)^{2}$ is no longer constant with height, due to the presence of wave-induced stress $\tau^{w}=\rho\left(u_{*}^{w}\right)^{2}$ associated to wave-induced motions. The sum of both wave-induced and turbulent contributions is however constant and equal to the square of the friction velocity $u_{*}^{2}$, defined as the normalized turbulent momentum flux on top of the WBL (defined as the SBL sub-layer where wave-induced stress is non-zero, e.g. Makin and Mastenbroek 1996)

$\left(u_{*}^{l}\right)^{2}(z)+\left(u_{*}^{w}\right)^{2}(z)=u_{*}^{2}$.

From this equation, we introduce the coupling coefficient

$\alpha_{c}(z)=\left[\frac{u_{*}^{w}(z)}{u_{*}}\right]^{2}$,

which quantifies the relative impact of wave-induced stress in the SBL. Equation 23 can then be rewritten as

$u_{*}^{l}=\left(1-\alpha_{c}\right)^{1 / 2} u_{*}$.

The second implication of the triple decomposition is that the TKE balance in the presence of wave-induced stress reads

$\left[\left(u_{*}^{w}\right)^{2}+\left(u_{*}^{l}\right)^{2}\right] \frac{\partial U}{\partial z}-\left(1+\beta_{2}\right) \frac{g H}{\rho C_{p} \theta_{h}}=\epsilon$.

With respect to the wall-bounded case (Eq. 1), the TKE balance now contains an additional term $\left(u_{*}^{w}\right)^{2} \partial U / \partial z$, describing the extraction of energy from the mean flow by its interaction with wave-induced stress. Equation 26 is a straightforward generalization of the Kudryavtsev et al. (2014) balance (derived in, e.g., Kudryavtsev and Makin 2004; Hara and Sullivan 2015) where stratification has been included (through the term $g H / C_{p} \theta_{h}$ ). In this balance, the presence of waves enhances TKE through the so-called wake production term, as found in numerical simulations over idealized sinusoidal waves Hara and Sullivan (2015) and over a breaking-wave field Suzuki et al. (2013).

Note that other balances could be considered. In particular, Janssen (1999) and Cifuentes-Lorenzen et al. (2018) consider that $\left(u_{*}^{w}\right)^{2} \partial U / \partial z$ acts directly as a source of wave energy (and hence does not appear in Eq. 26), leading to a decrease in TKE in the presence of windwaves. This balance describes TKE decrease observed very close to the surface in numerical simulations (Hara and Sullivan 2015). This region is not described by the Kudryavtsev et al. (2014) model, which is written in Cartesian coordinates, hence losing validity when approaching wave crests. 


\section{Input parameters}

\section{Wind-over-waves model}

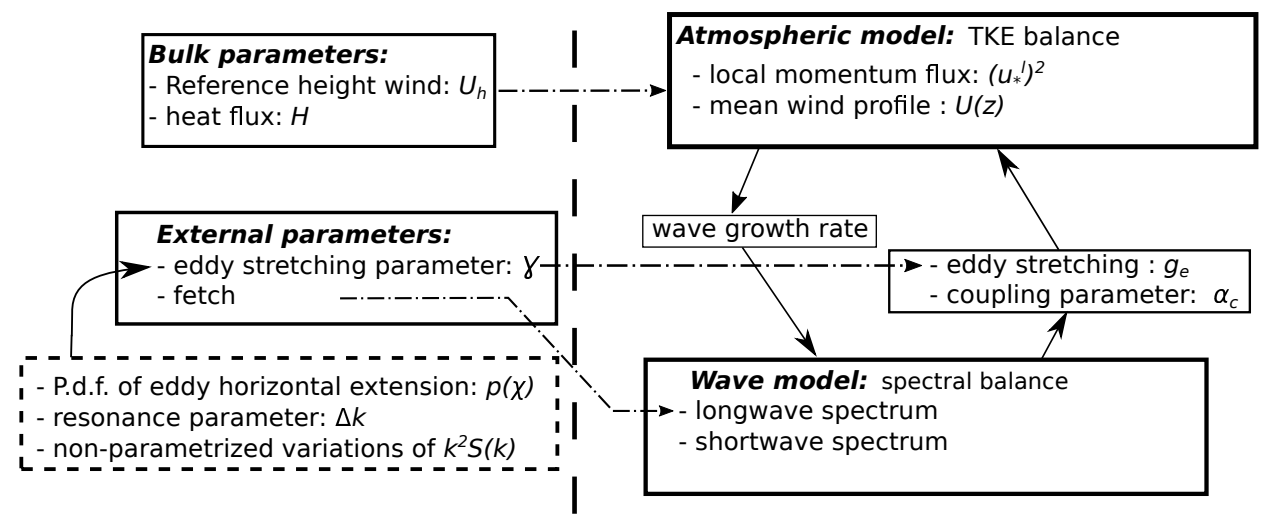

Fig. 2 Flow chart of the wind-over-waves model. The left panel shows the input parameters of the model and how they impact the different steps of model (dotted-dashed arrows). Note in particular the different quantities impacting the proxy eddy stretching parameter $\gamma$ (dashed box, Eq. 21). Right panel shows the structure of the wind-over-waves model. The equilibrium solution is obtained by iteratively solving the two model components (Eqs. 28) and 34), given a first guess for the momentum flux given by the bulk parameters

Using Eqs. 3 and 25 in Eq. 26, the TKE balance in dimensionless form reads

$-\left(1-\alpha_{c}\right)^{-1} \frac{\kappa z}{u_{*}^{l}} \frac{\partial U}{\partial z}+\left(1+\beta_{2}\right) \zeta+\frac{\kappa z}{\left(u_{*}^{l}\right)^{3}} \epsilon=0$.

The atmospheric component of the Kudryavtsev et al. (2014) wind-over-waves model is recovered for a neutral stratification (i.e. $\zeta \neq 0$ ). The standard TKE equation describing stratified turbulence in absence of waves is recovered in its dimensionless form (Eq. 5) for $\alpha_{c}=0$.

Using the expression for TKE dissipation Eq. 18 in Eq. 27 further yields

$$
\begin{aligned}
-\left(1-\alpha_{c}\right)^{-1} \frac{\kappa z}{u_{*}^{l}} & \frac{\partial U}{\partial z}+\left(1+\beta_{2}\right) \zeta \\
& +\frac{\left(u_{*}^{l}\right)^{3}}{(\kappa z)^{3}}\left(\frac{\partial U}{\partial z}\right)^{-3} f_{a}^{-1} g_{e}^{-1}=0
\end{aligned}
$$

where eddy anisotropy $f_{a}$ depends on stratification $\zeta$, and eddy stretching $g_{e}$ depends on $\gamma$, height $z$, and the wave spectrum (through Eqs. 19 and 21). Equation 28 can thus be solved for the dimensionless shear $\left[(\kappa z) / u_{*}^{l}\right] \partial U / \partial z$, given $\zeta, \alpha_{c}, f_{a}$, and $g_{e}$.

Wave-induced stress $\tau^{w}$, and thus $\alpha_{c}$, is required to solve Eq. 28. As presented in Kudryavtsev et al. (2014), the coupling parameter $\alpha_{c}$ describes not only waveinduced stress in the WBL resulting from the smooth deformation of the airflow above waves (losely called "form drag" in the following), but also stress induced by airflow separation events on top of breaking waves (Reul et al. 1999; Husain et al. 2019). For a given wave of wavelength $k$, both these effects act over a shallow atmospheric layer, up to heights $h(k) \sim 0.1 k^{-1}$ and $h_{a}(k) \sim 0.3 k^{-1}$ respectively. Note that this is at variance with eddy stretching, acting at greater heights (i.e. $h_{e} \sim 1 / k$, see Sect. 3$)$.

Furthermore, both form drag and airflow separation are, unlike eddy stretching, mostly confined to the short wind-waves range (waves of the order of $1 \mathrm{~m}$, following Plant 1982) and thus couple Eq. 28 to a stationary short wind-wave spectrum (described by Eq. 34 in Appendix 1 ). The full wind-wave spectrum is described, in the Kudryavtsev et al. (2014) model, as a superposition of the aforementioned short wind-waves part and a prescribed long-wave part, mostly governed by the degree of sea-state development (i.e. fetch and wave age, following Donelan et al. 1985; Elfouhaily et al. 1997, and discussed in Sect. 3.2). Details on both the wind-wave spectrum and the parametrization of the coupling coefficient can be found in Appendix 1.

The resulting wind-and-waves equilibrium matches atmospheric measurements (see Sect. 5.2) and wave measurements (Yurovskaya et al. 2013). It is not sensitive to variations in the long wind-wave spectrum (i.e. variations in fetch), since it does not contribute to form drag nor airflow separation.

\section{Momentum Fluxes Variability under Moderate Wind Speeds}

In this section, we first describe how the new physical mechanism (described in Sects. 2 and 3) can be incorporated in the wind-over-waves model described in Sect. 4 (Sect. 5.1). The resulting new wind-and-waves equilibrium is then compared to open-ocean measurements (Sect. 5.2). 


\subsection{The Coupled Model}

As summarized in Fig. 2, the coupled wind-over-waves model solves the TKE balance (28). The equation depends on the short wind-wave spectrum (through $\alpha_{c}$ ) obtained by solving a budget equation (Eq. 34 in Appendix 1). It also depends on the parameter $\gamma$ through $g_{e}$, introduced in Eq. 21. The coupled system is solved by iterations, given a $10-\mathrm{m}$ wind speed $U_{10}$, a heat flux $H$, fetch (for the prescribed long wind-wave spectrum), and the parameter $\gamma$. It returns a friction velocity on top of the WBL $\left(u_{*}\right)$, a mean wind profile $U(z)$, and a wind-wave spectrum $S(k)$, characterizing the windand-waves equilibrium. The inclusion of eddy stretching introduces a sensitivity of the wind-and-waves equilibrium to long wind-waves, absent in the Kudryavtsev et al. (2014) model and discussed below.

Note that even though Eq. 28 is valid for any stability condition, only unstable stratification conditions can be simulated by the coupled model (i.e. when $\alpha_{c}$ is determined recursively by solving Eq. 34 in Appendix $1)$. When the atmosphere is stably stratified, the TKE model yields unrealistically weak turbulence. It is out of the scope of the present paper to describe the coupled stably-stratified case, that would require the introduction of other physical processes such as gravity waves or a total kinetic energy balance (e.g. Zilitinkevich et al. 2008).

\subsection{Comparison to Experiments}

Near-surface momentum fluxes $\left(u_{*}^{2}\right)$ from the wind-overwaves model and its extension presented above were compared to measurements presented in Edson et al. (2013), compiling data obtained from different field campaigns in open sea. The data were collected $50 \mathrm{~km}$ off the southern Californian coast (Hristov et al. 2003), 10 $\mathrm{km}$ off the coast of Denmark (Mahrt et al. 1996), south of Martha's Vineyard (Edson et al. 2007), and on the northern wall of the Gulf Stream (Marshall et al. 2009), and were filtered to retain only young seas (i.e. with presumably no swell). This dataset covers a wide range of winds speeds (up to $25 \mathrm{~m} \mathrm{~s}^{-1}$ ) and stability conditions (stability parameter from -1.2 to 0.8 ). These observations are of particular relevance since they were used to calibrate the COARE parametrization (e.g. Fairall et al. 2003).

Figure 3 a shows the observed bin-averaged values of momentum fluxes as a function of the neutral 10$\mathrm{m}$ wind speed $\left(U_{10 N}\right.$, black dots). In the observations, neutral wind speed is obtained by applying a MOST stability correction function to the wind extrapolated

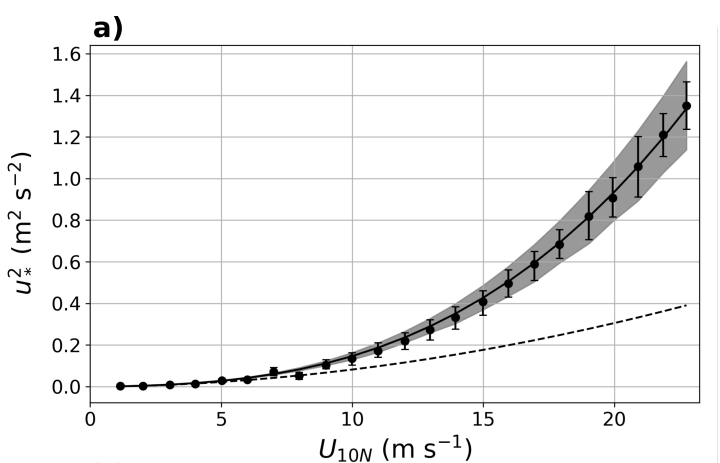

b)

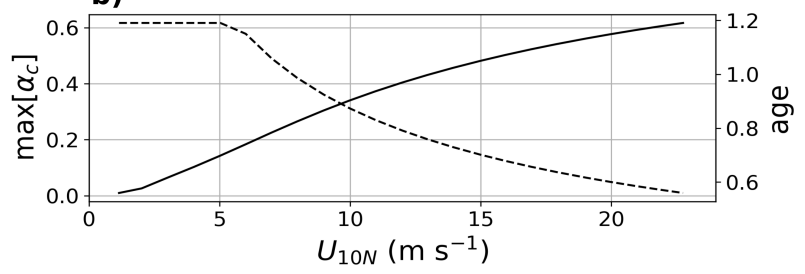

Fig. 3 (a) Surface momentum fluxes (divided by air density) vs neutral $10-\mathrm{m}$ wind speed. Dots indicate bin-averaged measurements from Edson et al. (2013), vertical error bars are the associated standard deviations, the dashed line is the model result without accounting for the impact of waves on the SBL, and the solid line is the model result with waveinduced stress. Grey shading is the range of values obtained varying eddy stretching around its neutral value of one (corresponding to $\gamma=0$ ). (b) Maximal (i.e. surface) coupling parameter $\alpha_{c}$ (solid line), and wave age (dashed line) vs 10$\mathrm{m}$ neutral wind speed. For the range of observed winds, the coupling parameter varies between 0 and 0.6

from direct measurements. It corresponds to the expected wind speed at equilibrium with the measured momentum flux in neutral stability conditions (e.g. Liu and Tang 1996). In the following, measurements will be compared with the wind-over-waves model in neutral conditions $(\zeta=0)$ leading to an eddy anisotropy factor $f_{a}=1$.

The solid line in Fig. 3a shows the equilibrium solution of the coupled model including wave-induced stress and without eddy stretching (i.e. $\gamma=0$ ), for a fetch of $100 \mathrm{~km}$. As expected from Kudryavtsev et al. (2014), the solution is in good agreement with observations. On the contrary, without wave-induced stress (i.e. $u_{*}^{w}=0$ or $\alpha_{c}=0$ ), the modelled momentum fluxes are smaller than those observed for wind speeds greater than about $10 \mathrm{~m} \mathrm{~s}^{-1}$ (dashed line in Fig. 3a). The effect of short waves on the SBL (through form drag and airflow separation, which increase TKE) is thus an essential physical process to explain the mean dependency of wind stress on $U_{10 N}$. In fact, for the considered range of wind values, the coupling coefficient $\alpha_{c}$ varies between 0 and 0.6 , and increases with wind speed (solid line in Fig. 3b, which shows the maximal coupling coeffi- 


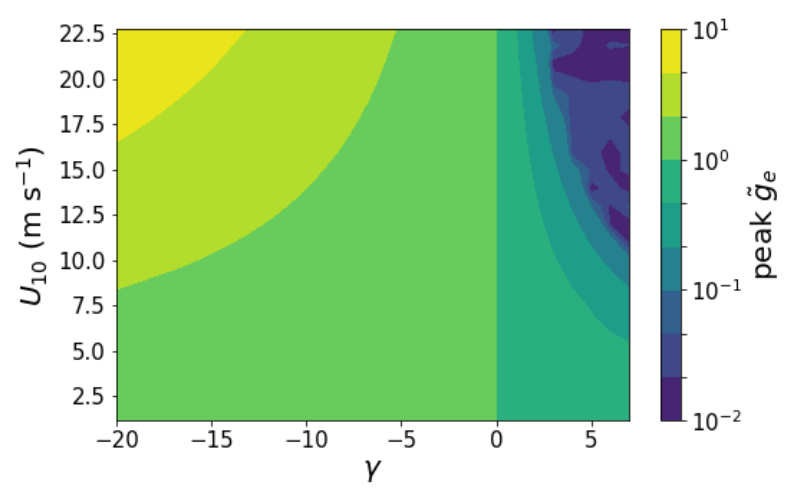

Fig. 4 Magnitude of eddy stretching $g_{e}$ at its spectral peak (from Eq. 21) as function of the coefficient $\gamma$ and 10-m wind

cient for a given wind, located very near the surface). For wind speeds above $15 \mathrm{~m} \mathrm{~s}^{-1}$, wave-induced stress is larger than the turbulent momentum stress (i.e. $\alpha_{c}>$ $0.5)$.

In the wind-over-waves model, to each value of $U_{10 N}$ corresponds a short wind-wave spectrum. Long windwaves depend on fetch and on wave age $\left(u_{*} / c_{p}\right.$, with $c_{p}$ the phase speed of the spectral peak wave component), following the parametrization of Donelan et al. (1985). The correspondence between $U_{10 N}$ and wave age in the model is shown in Fig. 3b (dashed line). The modelled range (between 0.6 and 1.2) is consistent with the range of observed values in Edson et al. (2013), which are interpreted as young seas. Note that even though there is a one-to-one correspondence between $U_{10 N}$ and wave age, there is no reason for such a relation between wave age and the near-surface momentum flux, which is discussed below by showing other sources of variability of momentum flux independent of wave age.

The momentum fluxes measurements exhibit a significant scatter (black error bars in Fig. 3a), which may be attributed to the influence of local processes on the wind-and-waves equilibrium (Edson et al. 2013). In the present work we investigate the possibility that this variability is caused by eddy stretching, through a change in the long wind--wave spectrum (and particularly 10-m waves), or through a change in the intensity of the modulation of short wind-waves by long windwaves. To this end, we use the simplified expression of eddy stretching (Eq. 21) in which a single parameter, $\gamma$, is varied as a proxy for these two effects. Variation of $\gamma$ between -20 and 8 yields the grey shading in Fig. 3. This corresponds to values of eddy stretching between $10^{-2}$ and $10^{1}$, as shown in Fig. 4. The range of variation in momentum flux resulting from the variation of eddy stretching covers the scatter that is observed in the data (compare shadings and error bars in Fig. 3). Note that the lower part of the grey shading in Fig. 3 corresponds to values of $g_{e}$ greater than one, and conversely. Other factors could be invoked to explain the scatter in the measurements, in particular fetch. To this end, fetch was varied in the coupled model between $10 \mathrm{~km}$ and $1000 \mathrm{~km}$, with fixed $\gamma$ in Eq. 21. The resulting variability was not sufficient to explain the observed scatter (not shown). This is consistent with the fact that fetch variations induce a change in eddy stretching at heights which do not significantly affect the surface momentum flux (as discussed in Sect. 3.2).

Wave-induced stress and eddy stretching were shown above to have a significant impact on surface momentum fluxes. Figure 5 further shows how both processes change TKE dissipation (Eq. 18) at different heights. As expected from the wind-over-waves model, TKE dissipation is enhanced when waves are included in the model (compare the dashed and the solid lines): the additional TKE production arising from wave-induced stress is locally balanced by an enhanced TKE dissipation. It is then interesting to focus on the sensitivity of TKE dissipation to variations of eddy stretching (grey shadings). The sensitivity of TKE dissipation to eddy stretching first decreases with height for heights below $5 \mathrm{~m}$ (compare grey shadings between Figs. 5a and 5b), and then increases with height above $5 \mathrm{~m}$ (compare grey shadings between Figs. 5c and 5d). This highlights two different causes of the sensitivity of TKE dissipation to changes in eddy stretching. Let us first recall that eddy stretching magnitude at a height $z\left(\tilde{g}_{e}\right)$ depends on the slope of waves whose wavenumber $k_{r}$ is such that $k_{r} \propto 1 / z$, called "resonant waves". This follows from Eqs. 12 and 21, where the resonant waves slope is $k_{r}^{2} S\left(k_{r}\right)$. Furthermore, the slope of wind-waves decreases with their wavenumber, since $S(k) \propto k^{-3}$ (in the "saturation range" of wind-waves, see, e.g. Phillips 1977, p. 148). Hence, near the surface (below $5 \mathrm{~m}$ ), the resonant waves (which are small) are not steep, and hence $\tilde{g}_{e}$ is close to one. At those heights, the observed sensitivity of TKE dissipation to eddy stretching thus results from the changes in the turbulent momentum flux $u_{*}^{l}$ (first factor in Eq. 18), caused by changes in TKE dissipation over the whole atmospheric column. As height increases, so does the slope of the resonant waves, and hence for heights above $5 \mathrm{~m}$, the sensitivity of TKE to eddy stretching results from $g_{e}$ being significantly different from one. Those two mechanisms show that the impact of long wind-waves on TKE dissipation can both directly and indirectly affect the whole atmospheric column.

Coming back to momentum fluxes, two effects can be invoked to explain their sensitivity to eddy stretching. First, as discussed above, stretching $g_{e}$ could di- 

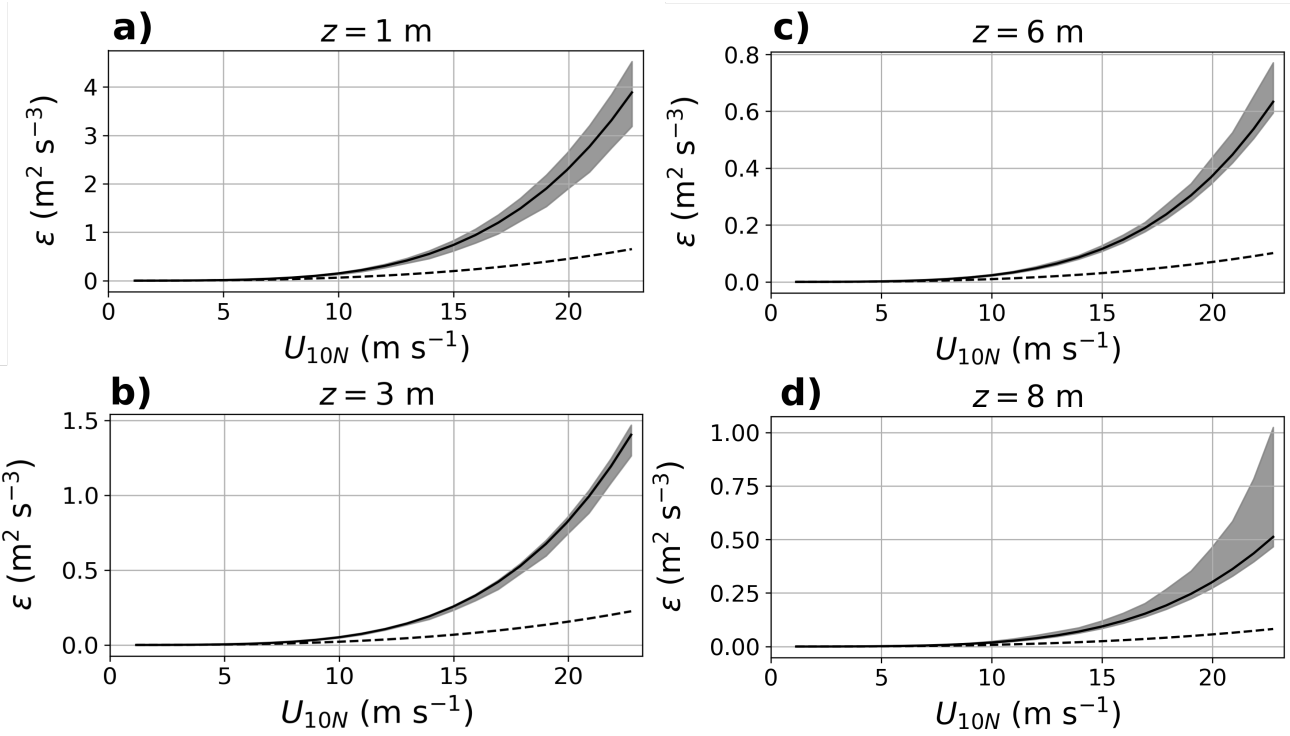

Fig. 5 TKE dissipation versus $10-\mathrm{m}$ wind speed at different heights. Note the differences in vertical-axis ranges. The dashed line is the model result without accounting for the impact of waves on the SBL and the solid line is the model result with wave-induced stress. Grey shading is the range of values obtained varying eddy stretching around its neutral value of one (corresponding to $\gamma=0$ )

rectly affect atmospheric turbulence through its (direct or indirect) effect on TKE dissipation $\epsilon$ (Eq. 18). Second, as the wave growth rate depends on atmospheric turbulence through $u_{*}^{l}$ (Eq. 35 in Appendix 1), the aforementioned modification, through a change in the wind-wave spectrum $S(k)$, could affect wave-induced stress $\tau^{w}$, ultimately leading to a change in atmospheric turbulence. Figure 6a shows three examples of modelled wind-wave spectra for the same value of $10-\mathrm{m}$ wind and different values of $\gamma$. It reveals that the short wind-wave spectrum on which wave-induced stress depends (i.e. $k$ of the order of $10^{3} \mathrm{~m}^{-1}$ ) is not significantly sensitive to variations in eddy stretching. To further assess if these variations are significant, we quantified their impact on atmospheric turbulence. Runs of an uncoupled version of the wind-over-waves model were performed and compared to coupled runs. A set of wave spectra were first computed by running a coupled wind-over-waves model with no eddy stretching $(\gamma=0)$ and different values of $U_{10 N}$. The uncoupled model was then run, meaning that the wind-wave dependent coupling variables $\alpha_{c}$ and $g_{e}$ in Eq. 28 were derived from the previous coupled runs at the same wind. The resulting momentum flux was then compared to the result of a coupled run with the same values of $\gamma$ and $U_{10 N}$. Over all the ranges of $U_{10 N}$ and $\gamma$, the relative difference between the momentum fluxes obtained from the coupled and uncoupled runs is lower than $0.4 \%$, as shown on Figure $6 \mathrm{~b}$, indicating that the variations in wind-wave spectrum due to eddy stretching do not significantly affect atmo- spheric turbulence. Thus, variations in eddy stretching do not significantly impact the short wind-wave spectrum, which was calibrated in Kudryavtsev et al. (2014) to fit observations (Yurovskaya et al. 2013). The short wind-wave spectrum is indeed determined by the coupling between low-level winds and short waves, occurring at heights where eddy stretching is negligible (i.e. at around $1 \mathrm{~m})$.

\section{Effects of Stability on the Surface Boundary Layer}

As discussed in Sect. 4, Kudryavtsev et al. (2014) did not include atmospheric stratification in their windover-waves model. However, the atmospheric turbulence model presented in Sect. 2.1 includes atmospheric stratification effects in the TKE balance. In Katul et al. (2011) it was further compared to Monin-Obukhov Similarity Theory (MOST). Based on dimensional arguments, MOST represents the impact of stratification on near surface momentum fluxes by means of a universal function (called MOST momentum function), determined from measurements. Katul et al. (2011) computed an analytical form of the MOST momentum function matching measurements. In this section we discuss how inclusion of waves into the Katul et al. (2011) framework changes the analytical MOST momentum function (Sect. 6.1), and how this compares to measurements (Sect. 6.2). 

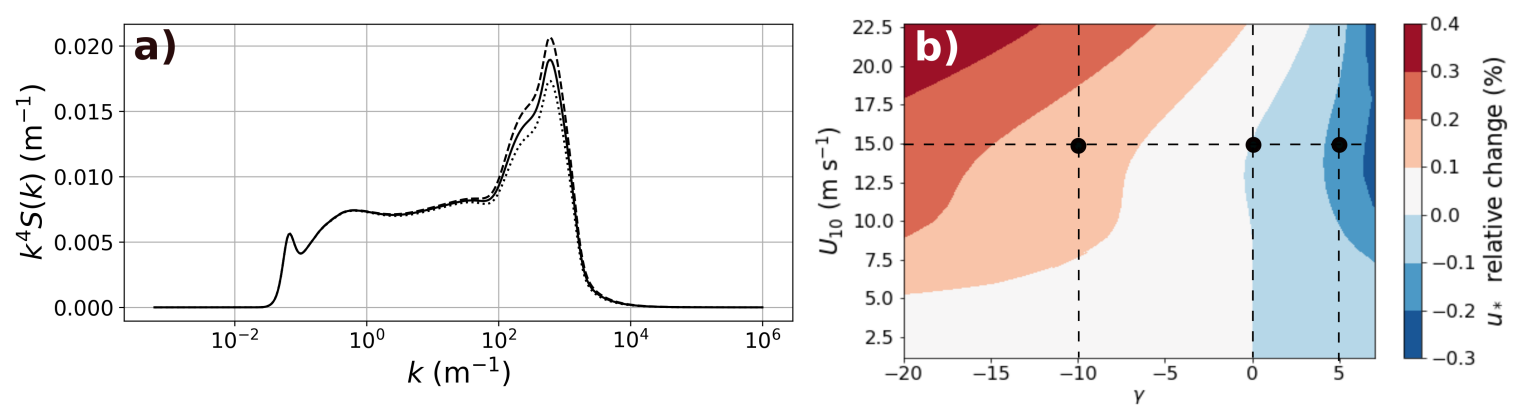

Fig. 6 (a) Modelled saturation spectrum $\left(k^{4} S(k)\right)$ for a 10-m wind of $15 \mathrm{~m} \mathrm{~s}^{-1}$ and an eddy stretching parameter $\gamma$ of -10 (dashed line), 0 (solid line), and 5 (dotted line); (b) Relative impact on momentum fluxes of a change in the wind-wave spectrum due to eddy stretching change represented as function of the coefficient $\gamma$ and $10-\mathrm{m}$ wind. Black dots indicate the three values of $U_{10}$ and $\gamma$ presented in (a)

6.1 Dependence of Monin-Obukhov Similarity Theory Momentum Function on Wind-Waves

One of the main outcomes of the Katul et al. (2011) work was to recover the MOST universal momentum function from the TKE budget (Eq. 5) and eddy anisotrop $\left(f_{a}\right)$. The authors recovered the O'KEYPS equation (Panofsky 1963; Businger 1988), which was originally derived based on heuristic and dimensional arguments to recover the empirical MOST momentum function over land (see Foken 2006), such as the Businger-Dyer function (Businger 1988, Eq. 39 in Appendix 2).

Defining the MOST momentum universal function (or dimensionless shear) as

$\phi_{m}=\frac{\kappa z}{u_{*}^{l}} \frac{\partial U}{\partial z}$

Eq. 28 can be rewritten as

$\left(1-\alpha_{c}\right)^{-1} \phi_{m}^{4}-\left(1+\beta_{2}\right) \zeta \phi_{m}^{3}=f_{a}^{-1} g_{e}^{-1}$,

assuming that $\left(1-\alpha_{c}\right) u_{*}^{4} \neq 0$.

The resulting equation reveals that $\phi_{m}$ depends on the wind-wave spectrum through $\alpha_{c}$ and $g_{e}$, unlike standard MOST which assumes that the universal momentum function only depends on $\zeta$. Furthermore, it extends the O'KEYPS equation and Katul et al. (2011) who considered the case of a flat boundary (i.e. $\alpha_{c}=0$ and $\left.g_{e}=1\right)$.

Equation 30 can be solved analytically (solutions, presented in the Supporting Information of Katul et al. 2011 , can be easily extended to the present case), yielding the MOST momentum function $\phi_{m}$. Note that in the following, we take $\alpha_{c}$ and $g_{e}$ as parameters of the model, unlike the coupled case where they are determined recursively by the wave model (Sect. 5.1). This allows exploration of all the range of stability conditions (in particular stable conditions $\zeta>0$ ).

The inverse of the solution of Eq. 30 as a function of $\zeta, \alpha_{c}$, and $g_{e}$ is shown in Fig. 7. The inverse of the MOST momentum function is of particular interest since it is proportional to the turbulent diffusion coefficient $\left(K\right.$, defined as $\left.\left(u_{*}^{l}\right)^{2}=K \partial_{z} U\right)$. Note first that the expected dependence of the turbulent diffusion coefficient with atmospheric stability is observed: turbulent diffusion is higher for an unstable atmosphere $(z / L<0)$ than for a stable atmosphere $(z / L>0)$. Second, an increase in wave-induced stress (i.e. in $\alpha_{c}$ in Fig. 7a) for a fixed stability induces an increase in turbulent diffusion. This is consistent with enhanced turbulent motions due to enhanced wake production. Third, eddy stretching greater (respectively lower) than one causes an increase (resp. a decrease) in turbulent diffusion, for a given stability (Fig. 7b). Since eddy stretching larger than one means reduced TKE dissipation, the observed increase in turbulent energy is consistent with an increase in the production term in the TKE equation, balanced by a constant energy-transfer term from wave motions and buoyancy, and a decreasing dissipation.

Figures 7a, b show how the sensitivity of atmospheric turbulence (i.e. the diffusion coefficient) to stability is modulated by wave-induced stress and eddy stretching. Figure 7 a reveals that increasing wave-induced stress causes an increase in this sensitivity. This is also the case for $g_{e}<1$, while eddy stretching larger than one causes a decrease in this sensitivity (Fig. 7b). The sensitivity of atmospheric turbulence to stability is an important feature since, as mentioned in the introduction, the first source of variability of turbulent momentum fluxes is atmospheric stability. These results indicate that short and long wind-waves play an important role in this variability.

\subsection{Comparison to Measurements}

Figure 8 shows the bin-averaged MOST momentum function as a function of stability for the measurements over open ocean from Edson et al. (2013) described 

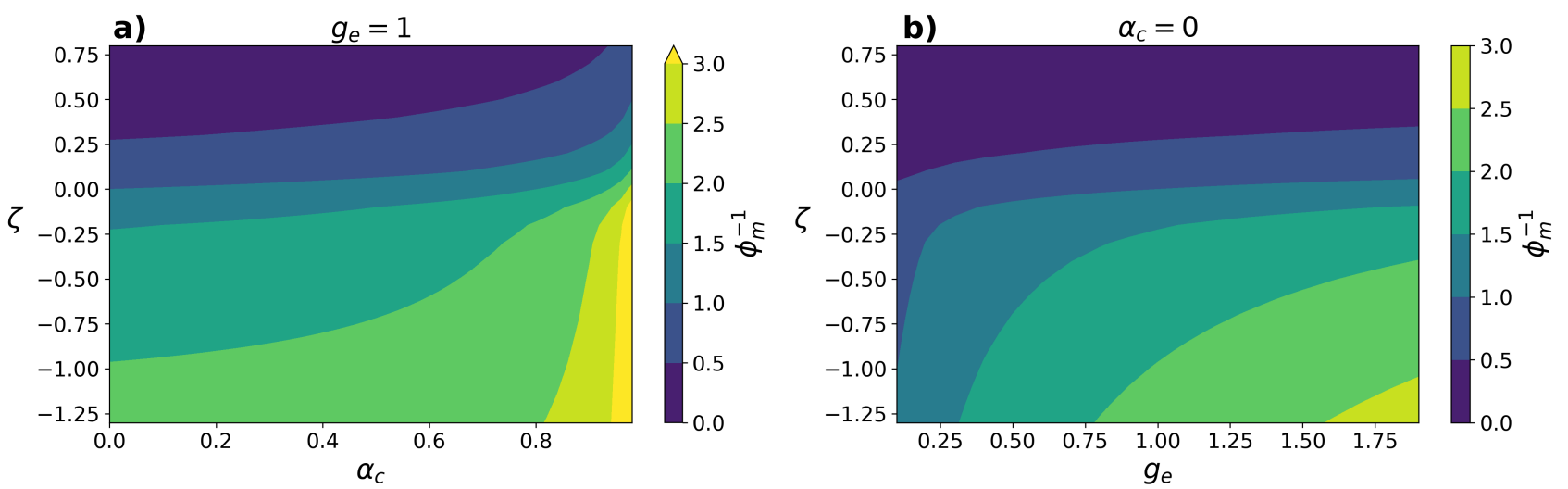

Fig. 7 Inverse universal momentum function $\phi_{m}^{-1}$ (proportional to turbulent diffusion) as a function of (a) stability and the coupling coefficient for eddy stretching set to one, (b) stability and eddy stretching for a coupling coefficient set to zero

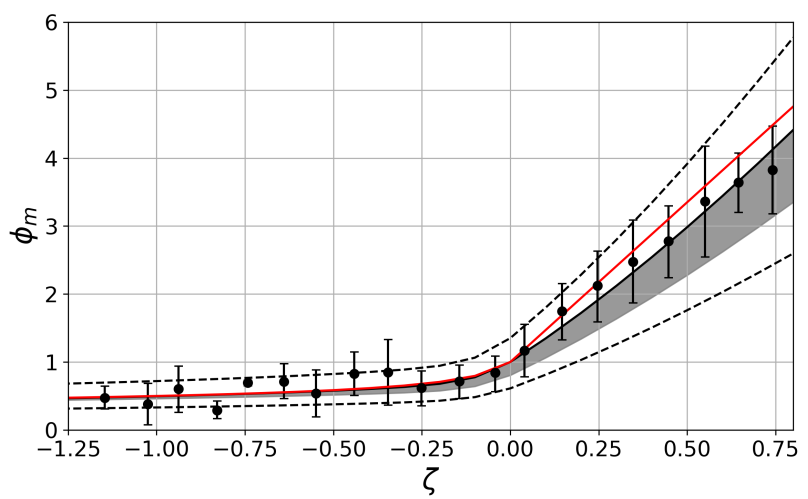

Fig. 8 Monin-Obukhov momentum universal function as a function of stability. Dots indicate bin-averaged measurements from Edson et al. (2013) and vertical error bars are the associated standard deviations. Red and black lines are the Businger-Dyer function (Businger 1988) and the present model result, respectively. Grey shading and dashed lines are the range of values obtained varying the wave coupling parameter and eddy stretching respectively

in Sect. 5.2 (black dots). The measurements were performed above or close to the expected height of the WBL (of the order of $5 \mathrm{~m}$ ) where MOST is expected to work (since wave-induced stress vanishes).

The solid black line in Fig. 8 represents the solution of Eq. 30 excluding the effect of waves (i.e. $\alpha_{c}=0$ and $\left.g_{e}=1\right)$ using the expression of eddy anisotropy $f_{a}$ from Katul et al. (2011) (Eq. 40 in Appendix 2). The values of $f_{a}$ are based on observed turbulent statistics over land (Kaimal et al. 1972). There is a good qualitative agreement between the model solution and the data, consistent with the measurements being at the expected height of the WBL. The Businger-Dyer function (red line) is also shown in Fig. 8 and fits similarly to the measurements, even though it differs from the Katul et al. (2011) solution in the stable case.
The scatter observed in the measurements (black error bars) contains contributions from both eddy covariance sampling uncertainties and variations in surface wave conditions. To investigate the contribution of the second effect on the scatter, Eq. 30 was solved for different values of the coupling coefficient $\alpha_{c}$ and eddy stretching $g_{e}$.

We first varied the coupling coefficient $\alpha_{c}$ to test the importance of wave-induced stress on atmospheric turbulence (through its effect on MOST) at heights above $5 \mathrm{~m}$. Even though it is usually assumed that this is not the case, airflow separation events on top of breaking waves could, for instance, extend higher in the SBL (see e.g. the numerical simulations of Suzuki et al. 2013). The range of variation of $\alpha_{c}$ (between 0 and 0.6 ) is inferred from the range obtained with the coupled windover-waves model in Sect. 5.2, and shown in Fig. 3b. It captures the range of observed short wind-wave conditions, neglecting atmospheric stratification effects. The resulting variation in modelled MOST functions (grey shading) shows that $\alpha_{c}$ might explain some scatter in the data, but the scatter is smaller than that found in observations. In particular, the data scatter in the stable case $(z / L>0)$ is not explained. Provided that waves are significant in explaining the measured scatter, this result indicates that sources of variability of $\phi_{m}$ other than short wind-waves variability should be investigated, such as eddy stretching.

Eddy stretching impacts TKE dissipation at heights above $5 \mathrm{~m}$ (being supported by 10-m waves, see Sect. 3.2), and can thus impact MOST momentum function (from Eq. 30). Variation of eddy stretching $g_{e}$ between 0.3 and 3 (dashed lines) covers the data scatter. This variation range is consistent with the one used in Sect. 5 (and shown in Fig. 4), showing that eddy stretching, and thus long wind-waves variability, seems to be able to explain the variability of MOST momentum func- 
tion. Note however that the variation range required to explain the observed scatter is smaller than the one required to explain the scatter of momentum fluxes under neutral conditions, and that this range could be even smaller if eddy covariance sampling uncertainties are deduced from the observed scatter.

Comparison of the wind-over-waves model with measurements thus shows that (i) observed stratification effects on MOST momentum function are consistent with the theoretical SBL model; (ii) Monin-Obukhov similarity theory quantities are less sensitive to the presence of wind-waves than momentum fluxes, as found in Hristov and Ruiz-Plancarte (2014) by only considering wave-induced motions within the momentum WBL.

\subsection{Dimensionless Dissipation}

Similarly to Fig. 5, we now investigate the sensitivity of TKE dissipation $\epsilon$ to stability. Following MOST, we define dimensionless dissipation $\phi_{\epsilon}$ as

$\phi_{\epsilon}=\frac{\kappa z \epsilon}{\left(u_{*}^{l}\right)^{3}}$,

which can be computed from Eq. 28 and 29 as

$\phi_{\epsilon}=\phi_{m}-\left(1+\beta_{2}\right) \zeta$.

The solid black line in Fig. 9 is the dimensionless dissipation computed with $\phi_{m}$ from Eqs. 30, excluding the effect of waves (i.e. $\alpha_{c}=0$ and $g_{e}=1$ ). As expected, there is a good agreement between this value and the Businger-Dyer function (red line). Values of $g_{e}$ larger (resp. smaller) than one cause a decrease (resp. an increase) in $\phi_{\epsilon}$, consistent with Eq. 18 (dashed lines, which correspond to $g_{e}=0.3$ and 3 respectively). Finally, as also shown in Fig. 5 for neutral conditions, an increase of wave-induced stress causes an increase of TKE dissipation (grey shading), consistent with the additional production term in the TKE balance equation. Overall, waves have a significant impact on the dimensionless TKE dissipation, consistent with previous studies (e.g. Cifuentes-Lorenzen et al. 2018).

In deriving the dimensionless equations (30) and (32), $u_{*}^{l}$ has been chosen as the normalizing velocity in the definition of $\phi_{m}, \phi_{\epsilon}$, and $\zeta$. This choice is physically sound, since in this case $\phi_{m}^{-2}$ is proportional to the turbulent diffusion. In measurements, however, it is difficult to disentangle wave-induced stresses from turbulent stresses (see e.g. Hristov et al. 2003), and the only measurable quantity could then be $u_{*}$, the total momentum flux. Hence, we must discuss the differences between choosing $u_{*}$ instead of $u_{*}^{l}$ as a normalizing velocity in MOST, i.e. by considering the following alternative forms of the universal momentum function,

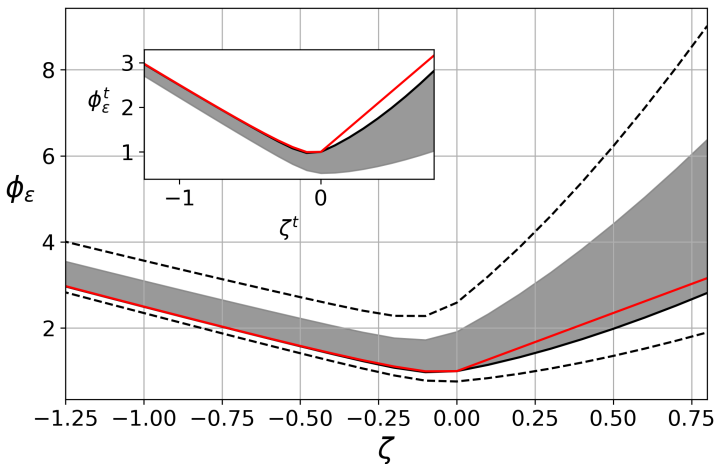

Fig. 9 Dimensionless TKE dissipation as a function of stability. Red line is the Businger-Dyer function (Businger 1988) and black line is the model result. Grey shading and dashed lines are the range of values obtained varying the wave coupling parameter or eddy stretching respectively. The inset shows the same quantities, but for a different choice of normalization velocity

dimensionless dissipation, and stability parameter

$\phi_{m}^{t}=\frac{\kappa z}{u_{*}} \frac{\partial U}{\partial z}, \quad \phi_{\epsilon}^{t}=\frac{\kappa z \epsilon}{u_{*}^{3}}, \quad \zeta^{t}=\frac{\kappa z g H}{\rho C_{p} \theta_{h} u_{*}^{3}}$.

First, there is no qualitative difference in the behaviour of $\phi_{m}^{t}$ with respect to $\phi_{m}$ (not shown). However, the behaviour of $\phi_{\epsilon}^{t}$, is qualitatively different from $\phi_{\epsilon}$. As shown in the inset of Fig. 9, an increase in wave-induced stress now causes a decrease in dimensionless dissipation (the grey shading is above the solid black line in the inset), inconsistent with the conclusions drawn earlier (in the main figure, the grey shading is below the black curve). This shows that MOST is sensitive to the choice of the normalization in the presence of waves. This could have important implications for the interpretation of measurements.

\section{Conclusion}

This study investigated the role of wind-waves and atmospheric stratification on atmospheric turbulence and momentum fluxes. The geometry of the ocean surface, resulting from the superposition of (periodic) surface wind-waves, is assumed to change the shape of energycontaining turbulent structures (conceptually viewed as attached eddies). Extending a wall-bounded turbulence model proposed by Katul et al. (2011) allowed the impact of this deformation on TKE dissipation to be modelled for a boundary whose height follows a wind-wave spectrum.

It was further argued that for an attached eddy of a given horizontal length scale, most of the deformation 
of its shape is due to surface waves with a similar wavelength. This was based on the assumption that the eddy deformation is caused by the surface wave periodically modulating the surface roughness induced by shorter waves, on a length scale resonant with that of the attached eddy. The overall result is a modification of TKE dissipation by long wind-waves (of the order of $10 \mathrm{~m}$ ), at heights above $5 \mathrm{~m}$, where the impact of wind-waves on atmospheric turbulence has been observed but was unexplained by wind-over-waves models.

The impact of the new mechanism on surface momentum fluxes was quantified by its inclusion in a windover-waves model (Kudryavtsev et al. 2014), which predicts a wind-and-waves equilibrium by coupling a TKE budget to a wind-wave energy budget. The wind-andwaves equilibrium, normally defined solely by $10-\mathrm{m}$ wind speed and atmospheric stability, is now also dependent on a single parameter linked to the long wind-wave spectrum and to the intensity of the modulation of short wind-waves roughness by long wind-waves (i.e. related to the deformation of attached eddies by windwaves). The variability observed over open ocean in both momentum flux (for a given 10-m wind speed) and MOST momentum function (for a given stability), was explained by variation of this parameter. If existent, the distortion of atmospheric eddies by the geometry of $10-\mathrm{m}$ wind-waves is thus an important process in the determination of surface momentum fluxes. This analysis also revealed that the sensitivity of MOST momentum functions to stability was impacted by the presence of both short and long wind-waves. Both processes should be included in momentum flux parametrizations.

wind-waves longer than $10 \mathrm{~m}$ were found to modify TKE dissipation at a height too high to impact surface momentum fluxes. However, if the assumptions of stationarity and horizontal homogeneity were relaxed (i.e. the SBL is no longer a constant-stress layer, which can occur, e.g., when the boundary layer height decreases significantly), their impact on TKE could significantly affect momentum fluxes in the whole surface layer. This process could then play an important role in the coupling of wind-waves with large scale atmospheric structures.

This theoretical work is based on the idea that surface waves are able to distort atmospheric eddies. However it does not rely on experimental evidence, and the expression of the distortion includes a free parameter. Katul and Manes (2014) linked the shape of the attached eddies to properties of the vertical turbulent velocity spectra. This link could be further investigated within open-ocean measurements in order to test the present theory. The proposed framework thus opens new paths for numerical and experimental investiga- tions of turbulence on top a realistic sea surface. Those would require the joint analysis of atmospheric vertical velocity and sea surface elevation/slope signals to infer the expected changes in turbulence spectral properties.

More generally, this work is a step towards a more precise description of multi-scale interactions within the WBL, linking the shape of large atmospheric structures with macroscopic properties of the surface wave field. By showing the importance of atmospheric eddy distortions for air-sea fluxes, we emphasize that this description is essential in order to advance our understanding of the wind-and-waves coupled system and to improve air-sea fluxes parametrizations.

Acknowledgements The code used to generate the figures is available upon request to the corresponding author. The authors thank the anonymous reviewers for the comments that helped improve the original manuscript. The authors wish to acknowledge V.N. Kudryavtsev for sharing the code and for the insightful help, and M.N. Bouin and S. Brumer for interesting discussions. AA was supported by DGA grant No D0456JE075 and French Brittany Regional Council. Finally, the authors also wish to acknowledge the support from ANR Caravele. 


\section{Appendix 1: Coupling Between Short Wind-Waves and Atmospheric Turbulence}

Details are provided on the coupling between short wind-waves and atmospheric turbulence, following the windover-waves model presented in Kudryavtsev et al. (2014) and references therein. The short wind-waves model is first described, and expressions for wave-induced stress are then presented.

Waves are described by their wavenumber $k$, frequency $\omega$, phase speed $c$, and direction of propagation $\psi$, and follow the dispersion relation $\omega^{2}=g k+T_{s w} k^{3}$ where $T_{s w}$ is the dynamical surface water tension. The wave field is specified by means of the directional spectrum $S^{d}(k, \psi)$. We also introduce the saturation spectrum $B(k, \psi)=k^{4} S^{d}(k, \psi)$, which will be used in the following.

As proposed by Kudryavtsev et al. (2014), the full wave spectrum can be defined as a composition of a shortwave spectrum $B_{s w}$ and a long-wave spectrum $B_{l w}$ (in this study, the fetch-dependent spectrum of Donelan et al. 1985 , is used). The weighted sum between $B_{l w}$ and $B_{s w}$ represents a wind-driven sea spectrum, without the presence of non-local waves (swell). It is in a one-to-one relation with the local atmospheric state. The shortwave spectrum is coupled to atmospheric turbulence through form drag, and further affects the momentum WBL through airflow separation stresses. The long-wave part is prescribed given some parameters (here spatial fetch).

The short-wave component $B_{s w}$ describes both gravity waves and parasitic capillary waves. The latter are generated on the forward face of shorter gravity waves (in the wavelength range 0.03-0.3 m), as they approach their maximum steepness, which, for longer gravity waves, would lead to breaking (Longuet-Higgins 1963).

The gravity short wind-wave spectrum results from a balance between wind forcing $(\beta)$, non-linear energy losses due to wave breaking (or generation of parasitic capillary waves for shorter waves), and generation of short waves by large breakers (or of parasitic capillary waves by steep and shorter waves, $Q_{b}$ ). The balance equation reads

$\beta_{v}(k, \psi) B(k, \psi)-B(k, \psi)\left(\frac{B(k, \psi)}{a}\right)^{n_{g}}+Q_{b}(k, \psi)=0$,

with $\beta_{v}(k, \psi)=\beta(k, \psi)-4 \nu k^{2} / \omega$ the effective growth rate (with $\nu$ air viscosity), and $a=2.2 \times 10^{-3}$ and $n_{g}=10$ two tuning constants fitted to observations (from Yurovskaya et al. 2013). Expression for the source term $Q_{b}$ can be found in Appendix A of Kudryavtsev et al. (2014).

The short parasitic capillary waves, corresponding to waves of wavelengths of $3 \times 10^{-4} \mathrm{~m}$ or less, follow the balance Eq. 34 without the wind input term, and with modified constants $a$ and $n_{g}$. For this range of waves for which wave breaking does not occur, the non-linear term is associated to a non-linear saturation of the wave spectrum.

Both equations are solved by iterations, given a wind forcing resulting from the WBL model (Eq. 28), and expressed as

$\beta(k, \psi)= \begin{cases}c_{\beta}\left\{\frac{u_{*}^{l}[h(k)]}{c}\right\}^{2} \cos \psi|\cos \psi| & \text { for } U[h(k)]>c \\ 0 & \text { for } U[h(k)]<c\end{cases}$

where $c_{\beta}=3 \times 10^{-2}$ is Plant's constant and $h(k)=0.1 k^{-1}$ is the inner region height. Note that since wind input depends on the ratio between friction velocity and wave phase speed, it is supported mostly by slow (and short) waves (Plant 1982).

To solve Eq. 28, wave-induced stress must be specified. Let $\tilde{T}$ and $\tilde{T}_{a}$ be the intensity of form drag and airflow separation induced by a wave component of wavenumber $k$. Both these effects act over a shallow atmospheric layer, up to heights $h(k) \sim 0.1 k^{-1}$ and $h_{a}(k) \sim 0.3 k^{-1}$, respectively (Kudryavtsev et al. 2014). We further assume, for simplicity, that form drag (respectively airflow separation) is constant up to $h$ (resp. $h_{a}$ ) and cancels for $z>h$ (resp. $z>h_{a}$ ). This yields the following expression for the total wave-induced stress

$$
\begin{aligned}
\left(u_{*}^{w}\right)^{2}(z)= & \int \tilde{T}(k) \operatorname{He}[h(k)-z] d k \\
& +\int \tilde{T}_{a}(k) \operatorname{He}\left[h_{a}(k)-z\right] d k
\end{aligned}
$$

where $\operatorname{He}(x)$ is the Heaviside step function $(\operatorname{He}(x)=1$ for $x>0$ and 0 otherwise). This expression couples the short wind-wave model (Eq. 34) to the SBL model (Eq. 28). 
Form drag describes the impact of the wind-to-waves energy transfer on atmospheric turbulence, and is expressed as:

$\tilde{T}(k)=\left\{\begin{array}{ll}\frac{c_{\beta}}{k} \frac{\rho_{w}}{\rho_{a}}\left\{u_{*}^{l}[h(k)]\right\}^{2} \int B(k, \psi)(\cos \psi)^{3} d \psi & \text { for } U[h(k)]>c \\ 0 & \text { for } U[h(k)]<c\end{array}\right.$,

where $\rho_{w}$ and $\rho_{a}$ are the density of water and air respectively.

Waves of wavelength greater than $0.3 \mathrm{~m}$ generate an additional stress due to airflow separation on top of breaking waves (Reul et al. 1999). The expression for airflow separation stress for a given wavenumber depends on wave-breaking statistics. However, following Phillips (1985), wave-breaking statistics can be related to wave energy dissipation (the second term from the left in Eq. 34). For waves in the equilibrium range, on top of which most of airflow separation events occur, the spectral balance (Eq. 34) is further assumed to be reduced only to a balance between wind input and dissipation. This results in the following expression for airflow separation for $u[h(k)]>c$ :

$T_{a}(k)=\frac{2 c_{d b} c_{\beta}}{a} h_{a}(k) k f_{g}(k)\left(\frac{U\left[h_{a}(k)\right]}{c}-1\right)^{2} \int B(k, \psi)(\cos \psi)^{5} d \psi$

where $f_{g}(k)$ is a cutoff function restricting airflow separation in the equilibrium range, and $c_{d b}$ is the local roughness on top of breaking crests, which has a mean value of 0.35 (see Kudryavtsev and Makin 2001). For $U[h(k)]<c$, airflow separation is assumed to vanish, which limits airflow separation to slow (short) waves (similar to form drag).

\section{Appendix 2: Expressions of Eddy Anisotropy and the Businger-Dyer Momentum Function}

The Businger-Dyer universal momentum function (Businger 1988), derived from the Kansas measurements, reads

$\phi_{m}^{B}(\zeta)=\left\{\begin{array}{ll}1+4.7 \zeta & \text { for } \zeta>0 \\ (1-15 \zeta)^{-1 / 4} & \text { for } \zeta<0\end{array}\right.$.

This empirical function was recovered by Katul et al. (2011), by considering an eddy anisotropy of the form

$f_{a}(\zeta)= \begin{cases}\left(1-\frac{0.38}{0.55}[1-\exp (15 \zeta)]\right)^{-1} & \text { for } \zeta \leq 0 \\ \left(1+\frac{1}{0.55} \zeta\right)^{-6} & \text { for } \zeta>0\end{cases}$

This expression was obtained from measurements of vertical turbulent velocity spectra (from Kaimal et al. 1972). 


\section{References}

Belcher S, Hunt J (1993) Turbulent shear flow over slowly moving waves. J Fluid Mech 251:109-148, DOI 10.1017/S0022112093003350

Belcher S, Hunt J (1998) Turbulent flow over hills and waves. Annu Rev Fluid Mech 30(1):507-538, DOI 10.1146/annurev.fluid.30.1.507

Brown GS (1979) Estimation of surface wind speeds using satellite-borne radar measurements at normal incidence. J Geophys Res Sol Ea 84(B8):3974-3978, DOI 10.1029/JB084iB08p03974

Businger J (1988) A note on the businger-dyer profiles. Boundary-Layer Meteorol 42(1-2):145-151, DOI 10.1007/BF00119880

Cifuentes-Lorenzen A, Edson JB, Zappa CJ (2018) Airsea interaction in the southern ocean: Exploring the height of the wave boundary layer at the air-sea interface. Boundary-Layer Meteorol 169(3):461-482, DOI 10.1007/s10546-018-0376-0

Donelan MA, Hamilton J, Hui W (1985) Directional spectra of wind-generated ocean waves. Philos Trans R Soc A 315(1534):509-562, DOI $10.1098 /$ rsta. 1985.0054

Dorman GE, Mollo-Christensen E (1973) Observation of the structure on moving gust patterns over a water surface (cat's paws). J Phys Oceanogr 3(1):120-132, DOI 10.1175/15200485(1973)003;0120:OOTSOM ¿2.0.CO;2

Drennan WM, Graber HC, Donelan MA (1999) Evidence for the effects of swell and unsteady winds on marine wind stress. J Phys Oceanogr 29(8):1853-1864, DOI 10.1175/15200485(1999)029¡1853:EFTEOS ¿2.0.CO;2

Dulov V, Kudryavtsev V, Bol'Shakov A (2013) A field study of whitecap coverage and its modulations by energy containing surface waves. In: Donelan MA, Drennan WM, Saltzman ES, Wanninkhof R (eds) Gas Transfer at Water Surfaces, American Geophysical Union, pp 187-192, DOI 10.1029/gm127p0187

Edson J, Crawford T, Crescenti J, Farrar T, Frew N, Gerbi G, Helmis C, Hristov T, Khelif D, Jessup A, Jonsson H, Li M, Mahrt L, McGillis W, Plueddemann A, Shen L, Skyllingstad E, Stanton T, Sullivan P, Sun J, Trowbridge J, Vickers D, Wang S, Wang Q, Weller R, Wilkin J, Williams AJ, Yue DKP, Zappa C (2007) The coupled boundary layers and air-sea transfer experiment in low winds. Bull Am Meteor Soc 88(3):341-356, DOI 10.1175/BAMS-88-3-341

Edson JB, Zappa CJ, Ware J, McGillis WR, Hare JE (2004) Scalar flux profile relationships over the open ocean. J Geophys Res Oceans 109(C8), DOI 10.1029/2003JC001960
Edson JB, Jampana V, Weller RA, Bigorre SP, Plueddemann AJ, Fairall CW, Miller SD, Mahrt L, Vickers D, Hersbach H (2013) On the exchange of momentum over the open ocean. J Phys Oceanogr 43(8):1589 1610, DOI 10.1175/JPO-D-12-0173.1

Elfouhaily T, Chapron B, Katsaros K, Vandemark D (1997) A unified directional spectrum for long and short wind-driven waves. J Geophys Res Oceans 102(C7):15,781-15,796, DOI 10.1029/97JC00467

Elperin T, Kleeorin N, Rogachevskii I, Zilitinkevich S (2002) Formation of large-scale semiorganized structures in turbulent convection. Phys Rev E 66(6):66,305-66,320, DOI 10.1103/PhysRevE.66.066305

Fairall C, Bradley EF, Hare J, Grachev A, Edson J (2003) Bulk parameterization of air-sea fluxes: Updates and verification for the coare algorithm. J Clim 16(4):571-591, DOI 10.1175/15200442(2003)016;0571:BPOASF ¿2.0.CO;2

Foken T (2006) 50 years of the monin-obukhov similarity theory. Boundary-Layer Meteorol 119(3):431-447, DOI 10.1007/s10546-006-9048-6

Geernaert G (1990) Bulk parameterizations for the wind stress and heat fluxes. In: Surface waves and fluxes, Springer, pp 91-172, DOI 10.1007/978-94-0092069-9_5

Gent P, Taylor P (1976) A numerical model of the air flow above water waves. J Fluid Mech 77(1):105-128, DOI 10.1017/S0022112076001158

Gioia G, Guttenberg N, Goldenfeld N, Chakraborty P (2010) Spectral theory of the turbulent meanvelocity profile. Phys Rev Lett 105(18):184,501, DOI 10.1103/PhysRevLett.105.184501

Grodsky SA, Kudryavtsev VN, Bentamy A, Carton JA, Chapron B (2012) Does direct impact of SST on short wind waves matter for scatterometry? Geophys Res Lett 39(12), DOI 10.1029/2012gl052091

Hara T, Belcher SE (2002) Wind forcing in the equilibrium range of wind-wave spectra. J Fluid Mech 470:223-245, DOI 10.1017/S0022112002001945

Hara T, Sullivan PP (2015) Wave boundary layer turbulence over surface waves in a strongly forced condition. J Phys Oceanogr 45(3):868-883

Hogstrom (1996) Review of some basic characteristics of the atmospheric surface layer. Boundary-Layer Meteorol 78(2):215-246, DOI 10.1007/BF00120937

Hristov T (2018) Mechanistic, empirical and numerical perspectives on wind-waves interaction. In: Procedia IUTAM, Elsevier B.V., vol 26, pp 102-111, DOI 10.1016/j.piutam.2018.03.010

Hristov T, Ruiz-Plancarte J (2014) Dynamic balances in a wavy boundary layer. J Phys Oceanogr 44(12):3185-3194, DOI 10.1175/JPO-D-13-0209.1 
Hristov T, Friehe C, Miller S (1998) Wave-coherent fields in air flow over ocean waves: Identification of cooperative behavior buried in turbulence. Phys Rev Lett 81(23):5245, DOI 10.1103/PhysRevLett.81.5245

Hristov T, Miller S, Friehe C (2003) Dynamical coupling of wind and ocean waves through wave-induced air flow. Nature 422(6927):55, DOI 10.1038 /nature01382

Husain N, Hara T, Buckley M, Yousefi K, Veron F, Sullivan P (2019) Boundary layer turbulence over surface waves in a strongly forced condition: Les and observation. J Phys Oceanogr (2019), DOI 10.1175/JPO-D-19-0070.1

Janssen PA (1999) On the effect of ocean waves on the kinetic energy balance and consequences for the inertial dissipation technique. J Phys Oceanogr 29(3):530-534, DOI 10.1175/15200485(1999)029;0530:OTEOOW ¿2.0.CO;2

Janssen PA, Viterbo P (1996) Ocean waves and the atmospheric climate. J Clim 9(6):1269-1287, DOI 10.1175/15200442(1996)009;1269:OWATAC ¿2.0.CO;2

Janssen PaEM (1989) Wave-Induced Stress and the Drag of Air Flow over Sea Waves. J Phys Oceanogr 19(6):745-754, DOI 10.1175/15200485(1989)019¡0745:WISATD ¿2.0.CO;2

Kaimal JC, Wyngaard J, Izumi Y, Coté O (1972) Spectral characteristics of surface-layer turbulence. Q J R Meteorol Soc 98(417):563-589, DOI 10.1002/qj.49709841707

Katul GG, Manes C (2014) Cospectral budget of turbulence explains the bulk properties of smooth pipe flow. Phys Rev E 90(6):063,008, DOI 10.1103/PhysRevE.90.063008

Katul GG, Konings AG, Porporato A (2011) Mean velocity profile in a sheared and thermally stratified atmospheric boundary layer. Phys Rev Lett 107(26):268,502, DOI 10.1103/PhysRevLett.107.268502

Kawamura H, Toba Y (1988) Ordered motion in the turbulent boundary layer over wind waves. J Fluid Mech 197:105-138, DOI 10.1017/S0022112088003192

Kitaigorodskii S (1973) Physics of air-sea interactions. Israël Program for scientific translations

Kudryavtsev V, Chapron B (2016) On growth rate of wind waves: impact of short-scale breaking modulations. J Phys Oceanogr 46(1):349-360, DOI 10.1175/JPO-D-14-0216.1

Kudryavtsev V, Makin V (2001) The impact of air-flow separation on the drag of the sea surface. Boundary-Layer Meteorol 98(1):155-171, DOI 10.1023/A:1018719917275
Kudryavtsev V, Myasoedov A, Chapron B, Johannessen JA, Collard F (2012) Imaging mesoscale upper ocean dynamics using synthetic aperture radar and optical data. J Geophys Res Oceans 117(C4):n/an/a, DOI 10.1029/2011jc007492

Kudryavtsev V, Chapron B, Makin V (2014) Impact of wind waves on the air-sea fluxes: A coupled model. J Geophys Res Oceans 46(2):1022-1037, DOI 10.1002/2013JC009412

Kudryavtsev VN, Makin VK (2004) Impact of Swell on the Marine Atmospheric Boundary Layer. J Phys Oceanogr 34(4):934-949, DOI 10.1175/15200485(2004)034;0934:IOSOTM ¿2.0.CO;2

Li D, Katul GG, Bou-Zeid E (2012) Mean velocity and temperature profiles in a sheared diabatic turbulent boundary layer. Phys Fluids 24(10):105,105, DOI 10.1063/1.4757660

Li Q, Gentine P, Mellado JP, McColl KA (2018) Implications of nonlocal transport and conditionally averaged statistics on monin-obukhov similarity theory and townsends attached eddy hypothesis. J Atmos Sci 75(10):3403-3431, DOI 10.1175/JAS-D-17-0301.1

Liu WT, Tang W (1996) Equivalent neutral wind. Jet Propulsion Laboratory, Tech rep

Longuet-Higgins MS (1963) The generation of capillary waves by steep gravity waves. J Fluid Mech 16(1):138-159, DOI 10.1017/S0022112063000641

Mahrt L, Vickers D, Howell J, Højstrup J, Wilczak JM, Edson J, Hare J (1996) Sea surface drag coefficients in the ris $\varnothing$ air sea experiment. J Geophys Res Oceans 101(C6):14,327-14,335, DOI 10.1029/96JC00748

Makin V, Kudryavtsev V (1999) Coupled sea surfaceatmosphere model: 1. wind over waves coupling. J Geophys Res Oceans 104(C4):7613-7623, DOI 10.1029/1999JC900006

Makin VK, Mastenbroek C (1996) Impact of waves on air-sea exchange of sensible heat and momentum. Boundary-Layer Meteorol 79(3):279-300, DOI 10.1007/BF00119442

Marshall J, Ferrari R, Forget G, Maze G, Andersson A, Bates N, Dewar W, Doney S, Fratantoni D, Joyce T, et al. (2009) The climode field campaign: Observing the cycle of convection and restratification over the gulf stream. Bull Am Meteor Soc 90(9):1337-1350, DOI 10.1175/2009BAMS2706.1

Marusic I, Monty JP (2019) Attached eddy model of wall turbulence. Annual Review of Fluid Mechanics 51:49-74, DOI 10.1146/annurev-fluid-010518-040427

Monin A, Yaglom A (1975) Statistical Fluid Mechanics. Translated from original russian 1967 version, MIT press, Cambridge, MA

Panofsky HA (1963) Determination of stress from wind and temperature measurements. Q J R Meteorol Soc 
89(379):85-94, DOI 10.1002/qj.49708937906

Phillips OM (1985) Spectral and statistical properties of the equilibrium range in wind-generated gravity waves. J Fluid Mech 156:505-531, DOI $10.1017 /$ S0022112085002221

Phillips OM (1977) The dynamics of the upper ocean. Cambridge University Press

Phillips W, Wu Z, Lumley J (1996) On the formation of longitudinal vortices in a turbulent boundary layer over wavy terrain. J Fluid Mech 326:321-341, DOI 10.1017/S0022112096008336

Pineau-Guillou L, Ardhuin F, Bouin MN, Redelsperger JL, Chapron B, Bidlot JR, Quilfen Y (2018) Strong winds in a coupled wave-atmosphere model during a north atlantic storm event: evaluation against observations. Q J R Meteorol Soc 144(711):317-332, DOI http://doi.org/10.1002/qj.3205

Plant WJ (1982) A relationship between wind stress and wave slope. J Geophys Res Oceans 87(C3):19611967, DOI 10.1029/JC087iC03p01961

Reul N, Branger H, Giovanangeli JP (1999) Air flow separation over unsteady breaking waves. Phys Fluids 11(7):1959-1961, DOI 10.1063/1.870058

Shimura T, Mori N, Takemi T, Mizuta R (2017) Longterm impacts of ocean wave-dependent roughness on global climate systems. J Geophys Res Oceans 122(3):1995-2011, DOI 10.1002/2016jc012621

Soloviev YP, Kudryavtsev V (2010) Wind-speed undulations over swell: Field experiment and interpretation. Boundary-Layer Meteorol 136(3):341-363, DOI 10.1007/s10546-010-9506-z

Suzuki N, Hara T, Sullivan PP (2013) Impact of breaking wave form drag on near-surface turbulence and drag coefficient over young seas at high winds. J Phys Oceanogr 43(2):324-343, DOI 10.1175/jpo-d12-0127.1

Townsend AA (1980) The structure of turbulent shear flow. Cambridge university press

Vandemark D, Edson JB, Chapron B (1997) Altimeter estimation of sea surface wind stress for light to moderate winds. J Atmos Ocean Technol 14(3):716-722, DOI 10.1175/15200426(1997)014;0716:AEOSSW ¿2.0.CO;2

Villas Boas AB, Ardhuin F, Ayet A, Bourassa MA, Chapron B, Brandt P, Cornuelle BD, Farrar JT, Fewings MR, Fox-Kemper B, et al. (2019) Integrated observations of global surface winds, currents, and waves: requirements and challenges for the next decade. Frontiers in Marine Science 6:425, DOI 10.3389/fmars.2019.00425

Yurovskaya M, Dulov V, Chapron B, Kudryavtsev V (2013) Directional short wind wave spectra derived from the sea surface photography. J Geophys Res
Oceans 118(9):4380-4394, DOI 10.1002/jgrc.20296

Zhang FW, Drennan WM, Haus BK, Graber HC (2009) On wind-wave-current interactions during the shoaling waves experiment. J Geophys Res Oceans 114(C1), DOI 10.1029/2008JC004998

Zilitinkevich S, Elperin T, Kleeorin N, Rogachevskii I, Esau I, Mauritsen T, Miles M (2008) Turbulence energetics in stably stratified geophysical flows: Strong and weak mixing regimes. Q J R Meteorol Soc 134(633):793-799, DOI 10.1002/qj.264 